\title{
THE G METHOD FOR HETEROGENEOUS ANISOTROPIC DIFFUSION ON GENERAL MESHES
}

\author{
LÉo Agélas ${ }^{1}$, Daniele A. Di Pietro ${ }^{1}$ and JÉrôme Droniou ${ }^{2}$
}

\begin{abstract}
In the present work we introduce a new family of cell-centered Finite Volume schemes for anisotropic and heterogeneous diffusion operators inspired by the MPFA L method. A very general framework for the convergence study of finite volume methods is provided and then used to establish the convergence of the new method. Fairly general meshes are covered and a computable sufficient criterion for coercivity is provided. In order to guarantee consistency in the presence of heterogeneous diffusivity, we introduce a non-standard test space in $H_{0}^{1}(\Omega)$ and prove its density. Thorough assessment on a set of anisotropic heterogeneous problems as well as a comparison with classical multi-point Finite Volume methods is provided.
\end{abstract}

Mathematics Subject Classification. 65N08, 65N12.

Received November 27, 2008.

Published online March 17, 2010.

\section{INTRODUCTION}

One of the key ingredients for the numerical solution of Darcy equations is the discretization of anisotropic heterogeneous elliptic terms [9]. Significant mathematical effort has therefore been devoted to finding consistent and robust Finite Volume (FV) discretizations of anisotropic heterogeneous elliptic terms on general meshes. Ideally, a method should (i) be consistent and coercive on general polyhedral meshes as well as robust with respect to the anisotropy and heterogeneity of the permeability tensor; (ii) yield well-conditioned linear systems for which optimal preconditioning strategies can be devised; (iii) have a narrow stencil, both to improve matrix sparseness and to reduce the communication in parallel implementations. The latter requirement speaks in favour of cell-centered methods. However, at present time, no unconditionally coercive and consistent compact stencil cell-centered method has been found. Indeed, although several symmetric methods display unconditional coercivity, they either entail severe mesh restrictions, as in [4], or exhibit very large stencils, as in [6,24].

The so-called Multi Point Flux Approximation (MPFA) methods have been independently introduced in the middle of the 90s by Aavatsmark et al. [2] and Edwards and Rogers [21]. The key idea is to obtain consistency on general meshes at the expense of a larger stencil while preserving the second order convergence of the classical two-points method. As mentioned, however, coercivity only holds under suitable conditions on both the mesh and the permeability tensor. The compact stencil MPFA L method has been proposed by Aavatsmark et al.

\footnotetext{
Keywords and phrases. Finite volume methods, heterogeneous anisotropic diffusion, MPFA, convergence analysis.

1 IFP, 1 \& 4 av. du Bois-Préau, 92852 Rueil-Malmaison Cedex, France. leo.agelas@ifp.fr;

daniele-antonio.di-pietro@ifp.fr

2 Université Montpellier 2, Institut de Mathématiques et Modélisation de Montpellier, CC 051, Place Eugène Bataillon, 34095 Montpellier Cedex 05, France. droniou@math.univ-montp2.fr
} 
$[3,5]$ as an improvement of the MPFA O method of [1] both in terms of stencil size and monotonicity properties. The convergence of the MPFA O method has been theoretically proved in [4] on two-dimensional quadrilateral grids and in $[7,8]$ on general two- and three-dimensional polyhedral meshes. In [25], the equivalence of multipoints methods with the lowest-order Mixed Finite Element method on matching triangular grids has been pointed out, and local coercivity conditions have been proposed. Other relatively inexpensive methods that deserve being mentioned are those developed in the Mimetic Finite Difference framework of [14-16], as well as the Hybrid Finite Volume scheme of [24] or the Mixed Finite Volume scheme of [19]. The analogies among the three classes of methods have been recently pointed out in [20]. Finally, a unified framework covering both FV and discontinuous Galerkin methods expressed in weak form has recently been introduced in [10] relying on the discrete functional analysis results of $[17,23]$.

In this work we propose a family of cell-centered schemes generalizing the MPFA L method. The key idea is to write the flux through a face as the weighted average of several L-type fluxes. A proper choice of the weights enhances the coercivity of the method, thereby improving its robustness with respect to the skewness of the mesh and to the anisotropy and heterogeneity of the permeability tensor. The provided convergence proof covers more general FV schemes expressed in terms of numerical fluxes and it is inspired by the techniques of Eymard, Gallouët and Herbin (see, e.g., $[22,24]$ ). The relevant requirements are weak flux consistency and coercivity. Convergence is then obtained using the discrete Sobolev embeddings and Rellich theorem proved in [24], Section 5. Unlike in [10], where methods in weak formulation are considered, we focus here on lower order methods in flux formulation. The interest of flux formulation is that (i) it provides a natural means to implement new methods in traditional two-point FV codes; (ii) it is more natural for a number of multi-points methods and (iii) it allows to further reduce the set of requirements for convergence (flux continuity, e.g., is not needed).

From a practical viewpoint, the proposed method is a good compromise between accuracy, robustness and computational cost. Indeed, the methods of $[14-16,20,24]$ require the introduction of additional face unknowns whose local elimination in terms of cell unknowns is, in general, not possible. While the resulting stability properties are highly appreciable, the increase in computational cost is often not affordable in large industrial simulations. Unconditionally coercive cell-centered methods like the ones of [24], Section 2.2, or of [6] have stencils extending to neighbours of neighbours, resulting in denser matrices and increased memory requirements. Also, in parallel implementations, two layers of ghost cells are needed to ensure communications among subdomains, resulting in heavily penalized scalability (message passing is still considered a bottle-neck when it comes to large industrial cases). More compact methods like the MPFA L method have up to now been based on (sophisticated) heuristics rather than on a extensive mathematical analysis. To the best of our knowledge, the present work contains the first rigorous convergence proof for the MPFA L method for general meshes and arbitrary heterogeneous anisotropic diffusion tensors. The aim of this paper is also to identify a minimal set of requirements for convergence and investigate the benefits of a deeper mathematical comprehension. The resulting MPFA G method outperforms the original version of $[3,5]$ on a number of representative test cases modelling some of the difficulties encountered in industrial simulations.

In order to avoid artificial regularity assumptions on the permeability tensor in the consistency proof, we have introduced a permeability dependent test space $\mathcal{Q}$ composed of continuous functions with possibly discontinuous gradients but continuous fluxes. This space is proved to be dense in $H_{0}^{1}(\Omega)$ following the ideas of [18]. To the best of our knowledge, the idea of selecting a problem-taylored test space as well as the density proof are new; also, the density results for $\mathcal{Q}$ is a general tool of independent interest.

The work is organized as follows: in Section 2 we present a general convergence study based on a minimal set of requirements; the analysis applies to fairly general FV methods expressed in terms of numerical fluxes. In Section 3 we present the G method, a generalization of the MPFA L scheme, and show that it fits in the analysis framework of Section 2; Section 4 is devoted to numerical tests. The performances of the proposed method are evaluated on a set of anisotropic and heterogeneous benchmarks on general meshes. A comparison against the MPFA O and L methods as well as against a variant of the symmetric unconditionally coercive scheme of [24], Section 2.2 (hereafter labeled Success), is provided. 


\section{Abstract Framework}

\subsection{Model problem}

Let $\Omega \subset \mathbb{R}^{d}, d \geq 1$, be an open bounded connected polygonal domain with boundary $\partial \Omega$ and let $P_{\Omega} \stackrel{\text { def }}{=}$ $\left\{\Omega_{i}\right\}_{i=1 \ldots N_{\Omega}}$ denote a finite partition of $\Omega$ into open connected disjoint polygonal subsets. Let $\Lambda$ be a symmetric tensor-valued function such that (s.t.) (i) $\Lambda_{\mid \Omega_{i}} \in\left[C^{2}\left(\overline{\Omega_{i}}\right)\right]^{d, d}$ for all $i=1 \ldots N_{\Omega}$ and (ii) there exist $0<\alpha_{0}<$ $\beta_{0}<+\infty$ s.t., for almost every (a.e.) $x \in \bar{\Omega}$, the spectrum of $\Lambda(x)$ is contained in $\left[\alpha_{0}, \beta_{0}\right]$. Consider the following problem:

$$
\begin{cases}\nabla \cdot(-\Lambda \nabla \bar{u})=f & \text { in } \Omega, \\ \bar{u}=0 & \text { on } \partial \Omega,\end{cases}
$$

where $f \in L^{r}(\Omega)$ with $r>1$ if $d=2$ and $r=\frac{2 d}{d+2}$ if $d>2$. The existence and uniqueness of a weak solution $\bar{u} \in H_{0}^{1}(\Omega)$ of problem (2.1) is a classical result.

Remark 2.1. Other standard types of boundary conditions can be considered. However, for easiness of presentation, we have preferred to consider the simpler homogeneous Dirichlet case.

In what follows, we shall provide the definition of a FV discretization of problem (2.1) as well as an analysis framework covering fairly general (possibly nonconforming) polygonal meshes.

Definition 2.1 (admissible family of discretizations). An admissible family of finite volume discretizations $\left\{\mathcal{D}_{n}\right\}_{n \in \mathbb{N}}$ is a triplet $\mathcal{D}_{n}=\left(\mathcal{T}_{n}, \mathcal{E}_{n}, \mathcal{P}_{n}\right)$, where

(i) $\mathcal{T}_{n}$ is a finite family of non-empty connected open disjoint subsets of $\Omega$ (the cells or control volumes) s.t. $\bar{\Omega}=\cup_{K \in \mathcal{T}_{n}} \bar{K}$ and $\mathcal{T}_{n}$ is compatible with $P_{\Omega}$ (each cell is contained in one element of the partition $P_{\Omega}$ ). For all $K \in \mathcal{T}_{n}$, we denote by $\mathrm{m}_{K}>0$ its $d$-dimensional measure (the volume) and let $\partial K \stackrel{\text { def }}{=} \bar{K} \backslash K$;

(ii) $\mathcal{E}_{n}$ is a finite family of subsets of $\bar{\Omega}$ (the faces) s.t., for all $\sigma \in \mathcal{E}_{n}, \sigma$ is a non-empty closed subset of a hyperplane of $\mathbb{R}^{d}$ with $(d-1)$-dimensional measure $\mathrm{m}_{\sigma}>0$ (the area), and s.t. the intersection of two different faces has zero $(d-1)$-dimensional measure. We assume that, for all $K \in \mathcal{T}_{n}$, there exists a subset $\mathcal{E}_{K}$ of $\mathcal{E}_{n}$ such that $\partial K=\cup_{\sigma \in \mathcal{E}_{K}} \sigma$. For a given $\sigma \in \mathcal{E}_{n}$, either $\mathcal{T}_{\sigma} \stackrel{\text { def }}{=}\left\{K \in \mathcal{T}_{n} \mid \sigma \in \mathcal{E}_{K}\right\}$ has exactly one element and then $\sigma \subset \partial \Omega$ (boundary face) or $\mathcal{T}_{\sigma}$ has exactly two elements (inner face); the sets of inner and boundary faces are denoted by $\mathcal{E}_{n, \text { int }}$ and $\mathcal{E}_{n \text {,ext }}$ respectively;

(iii) $\mathcal{P}_{n}=\left\{x_{K}\right\}_{K \in \mathcal{T}_{n}}$ is a family of points of $\Omega$ indexed by $\mathcal{T}_{n}$ (the cell centers) s.t. $x_{K} \in K$ and $K$ is star-shaped with respect to $x_{K}$. For all $K \in \mathcal{T}_{n}$ and for all $\sigma \in \mathcal{E}_{K}$ we denote by $d_{K, \sigma}$ the Euclidean distance between $x_{K}$ and the hyperplane supporting $\sigma$, and suppose that there exist $0<\varrho_{1}<+\infty$ and $0<\varrho_{2}<+\infty$ independent of $n$ s.t.

$$
\min _{K \in \mathcal{T}_{n}, \sigma \in \mathcal{E}_{K}} \frac{d_{K, \sigma}}{\operatorname{diam}(K)} \geq \varrho_{1}, \quad \min _{\sigma \in \mathcal{E}_{n, \text { int }}, \mathcal{T}_{\sigma}=\{K, L\}} \frac{\min \left(d_{K, \sigma}, d_{L, \sigma}\right)}{\max \left(d_{K, \sigma}, d_{L, \sigma}\right)} \geq \varrho_{2} .
$$

Figure 1(a) presents an example of admissible mesh in two space dimensions. Definition 2.1 also establishes refinement procedure to obtain admissible mesh families from an initial coarse grid. By items (ii) and (iii), and since $\frac{\mathrm{m}_{\sigma} d_{K, \sigma}}{d}$ is the measure of the convex hull $\triangle_{K, \sigma}$ of $x_{K}$ and $\sigma$ (see Fig. 1(b)), the following geometric relation holds:

$$
\forall K \in \mathcal{T}_{n}, \quad \sum_{\sigma \in \mathcal{E}_{K}} \mathrm{~m}_{\sigma} d_{K, \sigma}=d \mathrm{~m}_{K} .
$$

The size of the discretization is defined by $h_{\mathcal{D}_{n}} \stackrel{\text { def }}{=} \sup _{K \in \mathcal{T}_{n}} \operatorname{diam}(K)$. For all $K \in \mathcal{T}_{n}$ and $\sigma \in \mathcal{E}_{K}$, we denote by $\mathbf{n}_{K, \sigma}$ the unit vector normal to $\sigma$ outward to $K$. For all $K \in \mathcal{T}_{n}$, we set $\Lambda_{K} \stackrel{\text { def }}{=} \frac{1}{\mathrm{~m}_{K}} \int_{K} \Lambda(x) \mathrm{d} x$. The Euclidean norm of a vector $x \in \mathbb{R}^{n}$ will be denoted by $|x| \stackrel{\text { def }}{=} \sqrt{x \cdot x}$, while, for a matrice $A \in \mathbb{R}^{n, n},|A|$ will denote the norm 


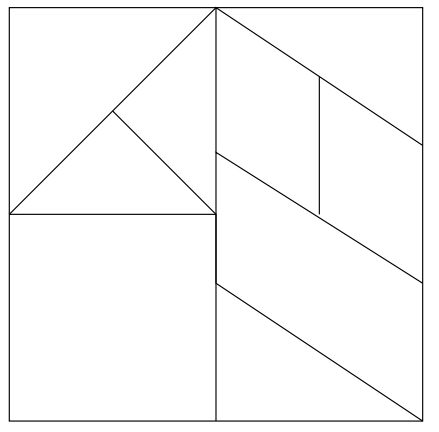

(a) Admissible mesh

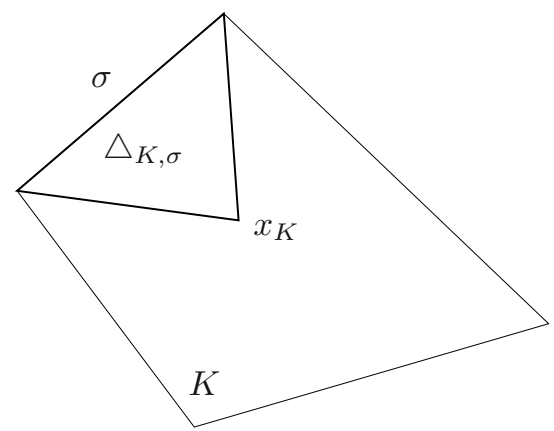

(b) Pyramid convex hull of $x_{K}$ and $\sigma$

Figure 1. Example of admissible mesh and pyramid convex hull of $x_{K}$ and $\sigma$ for $d=2$.

induced by the scalar product of $\mathbb{R}^{n}$, i.e., $|A| \stackrel{\text { def }}{=} \sup _{x \in \mathbb{R}^{d}} \frac{|A x|}{|x|}$. The vector space of bounded linear operators from $E$ to $F$ will be denoted by $\mathcal{L}(E ; F)$.

In what follows, when referring to a generic element $\mathcal{D}_{n}$ of an admissible family of discretizations $\left\{\mathcal{D}_{n}\right\}_{n \in \mathbb{N}}$, the subscript $n$ will be omitted to alleviate the notation whenever no ambiguity arises. The space of piecewise constant functions on $\mathcal{T}$ is defined as

$$
H_{\mathcal{T}}(\Omega) \stackrel{\text { def }}{=}\left\{v \in L^{2}(\Omega) \mid v_{\mid K} \in \mathbb{P}^{0}(K), \forall K \in \mathcal{T}\right\} .
$$

For all $v \in H_{\mathcal{T}}$ and for all $K \in \mathcal{T}, v_{K}$ will denote the (constant) value of $v$ on $K$, i.e., $v_{\mid K}(x)=v_{K}$ for all $x \in K$. In order to endow $H_{\mathcal{T}}$ with a discrete $H^{1}$ norm, let, for all $\sigma \in \mathcal{E}, I_{\sigma} \in \mathcal{L}\left(H_{\mathcal{T}}(\Omega) ; \mathbb{P}^{0}(\sigma)\right)$ denote a trace reconstruction operator s.t., for all $v \in H_{\mathcal{T}}(\Omega), I_{\sigma} v=0$ if $\sigma \in \mathcal{E}_{\text {ext }}$. We are now ready to define

$$
\|v\|_{\mathcal{T}, I} \stackrel{\text { def }}{=}\left(\sum_{K \in \mathcal{T}} \sum_{\sigma \in \mathcal{E}_{K}} \frac{\mathrm{m}_{\sigma}}{d_{K, \sigma}}\left|I_{\sigma} v-v_{K}\right|^{2}\right)^{1 / 2} .
$$

Remark 2.2. Let $\gamma_{\sigma} \in \mathcal{L}\left(H_{\mathcal{T}}(\Omega) ; \mathbb{P}^{0}(\sigma)\right)$ be s.t.

$$
\forall v \in H_{\mathcal{T}}(\Omega), \quad \begin{cases}\frac{\gamma_{\sigma} v-v_{K}}{d_{K, \sigma}}+\frac{\gamma_{\sigma} v-v_{L}}{d_{L, \sigma}}=0 & \text { if } \sigma \in \mathcal{E}_{\text {int }} \text { with } \mathcal{T}_{\sigma}=\{K, L\}, \\ \gamma_{\sigma} v=0 & \text { if } \sigma \in \mathcal{E}_{\text {ext }} .\end{cases}
$$

Then, for all $I_{\sigma} \in \mathcal{L}\left(H_{\mathcal{T}}(\Omega) ; \mathbb{P}^{0}(\sigma)\right)$ s.t., for all $v \in H_{\mathcal{T}}(\Omega), I_{\sigma} v=0$ if $\sigma \in \mathcal{E}_{\text {ext }}$,

$$
\forall v \in H_{\mathcal{T}}(\Omega), \quad\|v\|_{\mathcal{T}, \gamma} \leq\|v\|_{\mathcal{T}, I} .
$$

Set, for $\sigma \in \mathcal{E}_{\text {int }}$ with $\mathcal{T}_{\sigma}=\{K, L\}, g_{\sigma}(y) \stackrel{\text { def }}{=} \frac{\mathrm{m}_{\sigma}}{d_{K, \sigma}}\left|y-v_{K}\right|^{2}+\frac{\mathrm{m}_{\sigma}}{d_{L, \sigma}}\left|y-v_{L}\right|^{2}$. Inequality (2.4) is obtained by noticing that $\gamma_{\sigma} v$ minimizes $g_{\sigma}$ and that $\|v\|_{\mathcal{T}, \gamma}^{2}=\sum_{\sigma \in \mathcal{E}_{\text {int }}} g_{\sigma}\left(\gamma_{\sigma} v\right)+\sum_{\sigma \in \mathcal{E}_{\text {ext }}, \mathcal{T}_{\sigma}=\{K\}} \frac{\mathrm{m}_{\sigma}}{d_{K, \sigma}}\left|v_{K}\right|^{2}$.

In view of Remark 2.2 and of the special nature of $\gamma_{\sigma}$, the abridged notation $\|\cdot\|_{\mathcal{T}}$ will be used for $\|\cdot\|_{\mathcal{T}, \gamma}$ whenever possible. For all $K \in \mathcal{T}$ and for all $\sigma \in \mathcal{E}_{K}$, let $F_{K, \sigma} \in \mathcal{L}\left(H_{\mathcal{T}}(\Omega) ; \mathbb{P}^{0}(\sigma)\right)$ be a numerical flux function meant to approximate the diffusive flux flowing out $K$ through $\sigma$. For all $(u, v) \in\left[H_{\mathcal{T}}(\Omega)\right]^{2}$, define the bilinear form

$$
a_{\mathcal{T}}(u, v) \stackrel{\text { def }}{=} \sum_{K \in \mathcal{T}} \sum_{\sigma \in \mathcal{E}_{K}} F_{K, \sigma}(u)\left(I_{\sigma} v-v_{K}\right) .
$$


In what follows, we shall consider discretizations of $(2.1)$ of the form

$$
\text { Find } u \in H_{\mathcal{T}}(\Omega) \text { s.t. } a_{\mathcal{T}}(u, v)=\int_{\Omega} f v \text { for all } v \in H_{\mathcal{T}}(\Omega) \text {. }
$$

\subsection{Convergence analysis}

We introduce the discrete gradient reconstruction $\widetilde{\nabla}_{\mathcal{D}} \in \mathcal{L}\left(H_{\mathcal{T}}(\Omega) ;\left[H_{\mathcal{T}}(\Omega)\right]^{d}\right)$ s.t., for all $K \in \mathcal{T}$ and all $v \in H_{\mathcal{T}}(\Omega)$,

$$
\widetilde{\nabla}_{\mathcal{D}} v_{\mid K}=\frac{1}{\mathrm{~m}_{K}} \sum_{\sigma \in \mathcal{E}_{K}} \mathrm{~m}_{\sigma}\left(I_{\sigma} v-v_{K}\right) \mathbf{n}_{K, \sigma} .
$$

For all $v \in H_{\mathcal{T}}$ and for all $K \in \mathcal{T},\left(\widetilde{\nabla}_{\mathcal{D}} v\right)_{K}$ will denote the (constant) value of $\widetilde{\nabla}_{\mathcal{D}} v$ on $K$, i.e., $\widetilde{\nabla}_{\mathcal{D}} v_{\mid K}(x)=$ $\left(\widetilde{\nabla}_{\mathcal{D}} v\right)_{K}$ for all $x \in K$. Equation (2.3) together with Cauchy-Schwarz inequality yield

$$
\left\|\widetilde{\nabla}_{\mathcal{D}} v\right\|_{\left[L^{2}(\Omega)\right]^{d}} \leq \sqrt{d}\|v\|_{\mathcal{T}, I} \quad \forall v \in H_{\mathcal{T}}(\Omega) .
$$

The next result can proved following [24], Section 5.1.2 (indeed, the discrete norms considered are equivalent under the mesh regularity assumptions of Def. 2.1):

Lemma 2.1 (discrete Sobolev embeddings). Let $\mathcal{D}$ be an element of a family of discretizations matching Definition 2.1. Let $q \in[1,+\infty)$ if $d=2$, and $q \in[1,2 d /(d-2)]$ if $d>2$. Then, there exists $C_{1}>0$ depending on $\Omega, q, \varrho_{1}$ and $\varrho_{2}$ but not on $n$ s.t.

$$
\|u\|_{L^{q}(\Omega)} \leq C_{1}\|u\|_{\mathcal{T}} \quad \forall u \in H_{\mathcal{T}}(\Omega)
$$

Owing to Remark 2.2, the following theorem can easily be deduced from (2.7) using techniques of [24], Lemmata 5.6-5.7:

Lemma 2.2 (discrete Rellich theorem). Let $\left\{\mathcal{D}_{n}\right\}_{n \in \mathbb{N}}$ be a sequence of admissible discretizations matching Definition 2.1 and s.t. $h_{\mathcal{D}_{n}} \rightarrow 0$ as $n \rightarrow \infty$, and let $\left\{v_{n}\right\}_{n \in \mathbb{N}}$ be a sequence of $H_{\mathcal{T}_{n}}(\Omega)$ s.t. there exists $C>0$ with $\left\|v_{n}\right\|_{\mathcal{T}_{n}, I} \leq C$ for all $n \in \mathbb{N}$. Then, there exist a subsequence of $\left\{v_{n}\right\}_{n \in \mathbb{N}}$ and a function $\widetilde{v} \in H_{0}^{1}(\Omega)$ s.t., as $n \rightarrow \infty$, (i) $v_{n} \rightarrow \widetilde{v}$ in $L^{q}(\Omega)$ for all $q \in\left[1,2 d /(d-2)\right.$ ) (and weakly in $L^{2 d /(d-2)}(\Omega)$ if $d>2$ ); (ii) $\left\{\widetilde{\nabla}_{\mathcal{D}_{n}} v_{n}\right\}_{n \in \mathbb{N}}$ weakly converges to $\nabla \widetilde{v}$ in $\left[L^{2}(\Omega)\right]^{d}$.

The assumptions yielding convergence of the finite volume scheme are gathered in the following

Assumption 2.1. Let $\left\{\mathcal{D}_{n}\right\}_{n \in \mathbb{N}}$ be a family of discretizations matching Definition 2.1 and s.t. $h_{\mathcal{D}_{n}} \rightarrow 0$ as $n \rightarrow \infty$. We suppose that

$(\mathrm{P} 1) \mathfrak{D}$ is a dense subspace of $H_{0}^{1}(\Omega)$ s.t. $\mathfrak{D} \subset C_{0}(\bar{\Omega}) \cap C^{2}\left(P_{\Omega}\right)$, where $C^{2}\left(P_{\Omega}\right)$ is the set of functions whose restriction to $\Omega_{i}, i=1 \ldots N_{\Omega}$, is $C^{2}\left(\overline{\Omega_{i}}\right)$ and $C_{0}(\bar{\Omega})$ denotes the space of continuous functions which vanish on $\partial \Omega$. For all $\varphi \in \mathfrak{D}$, we denote by $\varphi_{\mathcal{T}_{n}}$ the element of $H_{\mathcal{T}_{n}}(\Omega)$ s.t., for all $K \in \mathcal{T}_{n}, \varphi_{\mathcal{T}_{n} \mid K}=\varphi\left(x_{K}\right)$;

(P2) $a_{\mathcal{T}_{n}}$ is uniformly coercive, i.e., there is $\gamma_{1}>0$ independent of $n$ s.t.

$$
\forall v \in H_{\mathcal{T}_{n}}(\Omega), \quad a_{\mathcal{T}_{n}}(v, v) \geq \gamma_{1}\|v\|_{\mathcal{T}_{n}, I}^{2}
$$

(P3) the numerical fluxes are weakly consistent on $\mathfrak{D}$, i.e., for all $\varphi \in \mathfrak{D}$,

$$
\epsilon_{\mathcal{D}_{n}}(\varphi) \stackrel{\text { def }}{=}\left(\sum_{K \in \mathcal{T}_{n}} \sum_{\sigma \in \mathcal{E}_{K}} \frac{d_{K, \sigma}}{\mathrm{m}_{\sigma}}\left|F_{K, \sigma}\left(\varphi_{\mathcal{T}_{n}}\right)-\mathrm{m}_{\sigma}\langle\Lambda \nabla \varphi\rangle_{K} \cdot \mathbf{n}_{K, \sigma}\right|^{2}\right)^{\frac{1}{2}} \rightarrow 0 \text { as } n \rightarrow \infty,
$$

where, for all $K \in \mathcal{T}$ and for all $\Phi \in L^{1}(K)$, we have set $\langle\Phi\rangle_{K} \stackrel{\text { def }}{=} \int_{K} \Phi(x) \mathrm{d} x / \mathrm{m}_{K}$. For vectorial functions, the notation has to be intended component-wise. 
Remark 2.3. Owing to (2.3), Property (P3) holds for strongly consistent numerical fluxes, for which there is $C_{2}$ independent of $n$ s.t., for all $\varphi \in \mathfrak{D}$,

$$
\forall K \in \mathcal{T}_{n}, \forall \sigma \in \mathcal{E}_{K}, \quad\left|F_{K, \sigma}\left(\varphi_{\mathcal{T}_{n}}\right)-\mathrm{m}_{\sigma}\langle\Lambda \nabla \varphi\rangle_{K} \cdot \mathbf{n}_{K, \sigma}\right| \leq C_{2} \mathrm{~m}_{\sigma} h_{\mathcal{D}_{n}} .
$$

Indeed, owing to (2.3), (2.9) implies $\epsilon_{\mathcal{D}_{n}}(\varphi) \leq C_{2} \sqrt{d \mathrm{~m}_{\Omega}} h_{\mathcal{D}_{n}}$, whence (P3).

Remark 2.4. Finite Volume methods are usually conservative, i.e., for all $v \in H_{\mathcal{T}_{n}}(\Omega)$ and all $\sigma \in \mathcal{E}_{n \text {,int }}$ with $\mathcal{T}_{\sigma}=\{K, L\}, F_{K, \sigma}(v)+F_{L, \sigma}(v)=0$. This property is not required to prove Theorem 2.1 below. However, it is usually needed in the proof of (P2) or even in the definition of the numerical fluxes. When conservativity holds, problem (2.5) is equivalent to the (more classical) FV formulation:

$$
\text { Find } u \in H_{\mathcal{T}}(\Omega) \text { s.t. }-\sum_{\sigma \in \mathcal{E}_{K}} F_{K, \sigma}(u)=\int_{K} f(x) \mathrm{d} x \text { for all } K \in \mathcal{T},
$$

and the bilinear form $a_{\mathcal{T}}$ does not depend on the choice of the trace operators $\left\{I_{\sigma}\right\}_{\sigma \in \mathcal{E}}$.

Proposition 2.1 (asymptotic stability of the interpolator). Under Assumption 2.1, for all $\varphi \in \mathfrak{D}$,

$$
\left\|\varphi_{\mathcal{T}}\right\|_{\mathcal{T}, I} \leq \frac{1}{\gamma_{1}}\left(\epsilon_{\mathcal{D}}(\varphi)+\beta_{0} \sqrt{d}|\varphi|_{H^{1}(\Omega)}\right) .
$$

Proof. Let $\varphi \in \mathfrak{D}$. Owing to (2.6), for all $v \in H_{\mathcal{T}}(\Omega)$, the following integration by parts formula holds:

$$
\begin{aligned}
\sum_{K \in \mathcal{T}_{n}} \sum_{\sigma \in \mathcal{E}_{K}} \mathrm{~m}_{\sigma}\langle\Lambda \nabla \varphi\rangle_{K} \cdot \mathbf{n}_{K, \sigma}\left(I_{\sigma} v-v_{K}\right) & =\sum_{K \in \mathcal{T}_{n}} \int_{K} \Lambda(x) \nabla \varphi(x) \cdot\left(\frac{1}{\mathrm{~m}_{K}} \sum_{\sigma \in \mathcal{E}_{K}} \mathrm{~m}_{\sigma} \mathbf{n}_{K, \sigma}\left(I_{\sigma} v-v_{K}\right)\right) \mathrm{d} x \\
& =\sum_{K \in \mathcal{T}_{n}} \int_{K} \Lambda(x) \nabla \varphi(x) \cdot\left(\widetilde{\nabla}_{\mathcal{D}} v\right)_{K} \mathrm{~d} x=\int_{\Omega} \Lambda(x) \nabla \varphi(x) \cdot \widetilde{\nabla}_{\mathcal{D}} v(x) \mathrm{d} x .
\end{aligned}
$$

The above result together with (P2), Cauchy-Schwarz inequality and (2.7) yield

$$
\begin{gathered}
\gamma_{1}\left\|\varphi_{\mathcal{T}}\right\|_{\mathcal{T}, I}^{2} \leq a_{\mathcal{T}}\left(\varphi_{\mathcal{T}}, \varphi_{\mathcal{T}}\right)=\sum_{K \in \mathcal{T}} \sum_{\sigma \in \mathcal{E}_{K}} \sqrt{\frac{d_{K, \sigma}}{\mathrm{m}_{\sigma}}}\left[F_{K, \sigma}\left(\varphi_{\mathcal{T}}\right)-\mathrm{m}_{\sigma}\langle\Lambda \nabla \varphi\rangle_{K} \cdot \mathbf{n}_{K, \sigma}\right] \sqrt{\frac{\mathrm{m}_{\sigma}}{d_{K, \sigma}}}\left(I_{\sigma} \varphi_{\mathcal{T}}-\varphi_{K}\right) \\
\quad+\int_{\Omega} \Lambda(x) \nabla \varphi(x) \cdot \widetilde{\nabla}_{\mathcal{D}} \varphi_{\mathcal{T}}(x) \mathrm{d} x \\
\leq \epsilon_{\mathcal{D}}(\varphi)\left\|\varphi_{\mathcal{T}}\right\|_{\mathcal{T}, I}+\beta_{0}|\varphi|_{H^{1}(\Omega)}\left\|\widetilde{\nabla} \varphi_{\mathcal{T}}\right\|_{\left[L^{2}(\Omega)\right]^{d}} \leq\left(\epsilon_{\mathcal{D}}(\varphi)+\beta_{0} \sqrt{d}|\varphi|_{H^{1}(\Omega)}\right)\left\|\varphi_{\mathcal{T}}\right\|_{\mathcal{T}, I} .
\end{gathered}
$$

Lemma 2.3 (well-posedness). Assume that Assumption 2.1 holds. Then, problem (2.5) is well-posed for each $n \in \mathbb{N}$, and the solutions $u_{n} \in H_{\mathcal{D}_{n}}(\Omega)$ satisfy the stability estimate

$$
\left\|u_{n}\right\|_{\mathcal{T}_{n}, I} \leq \frac{C_{1}}{\gamma_{1}}\|f\|_{L^{r}(\Omega)} .
$$

Proof. The solvability of problem (2.5) stems from (P2), which guarantees the inversibility of the corresponding linear system for each $n$. Using (P2), Hölder's inequality, Lemma 2.1 and Remark 2.2, it is inferred from the integrability assumptions on $f$ that

$$
\gamma_{1}\left\|u_{n}\right\|_{\mathcal{T}_{n}, I}^{2} \leq a_{\mathcal{T}_{n}}\left(u_{n}, u_{n}\right)=\int_{\Omega} f u \mathrm{~d} x \leq\|f\|_{L^{r}(\Omega)}\left\|u_{n}\right\|_{L^{r^{\prime}}(\Omega)} \leq C_{1}\|f\|_{L^{r}(\Omega)}\left\|u_{n}\right\|_{\mathcal{T}_{n}, I},
$$

where we have set $r^{\prime} \stackrel{\text { def }}{=} \frac{r}{r-1}$. 
Theorem 2.1 (convergence). Let $\left\{\mathcal{D}_{n}\right\}_{n \in \mathbb{N}}$ be a family of discretizations matching Assumption 2.1 and s.t. $h_{\mathcal{D}_{n}} \rightarrow 0$ as $n \rightarrow \infty$. Then, as $n \rightarrow \infty$, the sequence of discrete solutions of problem $(2.5)$, say $\left\{u_{n}\right\}_{n \in \mathbb{N}}$, converges to the solution $\bar{u}$ of $(2.1)$ in $L^{q}(\Omega)$ for all $q \in\left[1,2 d /(d-2)\right.$ ) (and weakly in $L^{2 d /(d-2)}(\Omega)$ if $\left.d>2\right)$.

Proof. Owing to the stability estimate (2.11) together with Lemma 2.2, there is $\widetilde{u} \in H_{0}^{1}(\Omega)$ s.t., up to a subsequence, (i) $\left\{u_{n}\right\}_{n \in \mathbb{N}}$ converges to $\widetilde{u}$ in $L^{q}(\Omega)$ for all $q \in\left[1,2 d /(d-2)\right.$ ) (and weakly in $L^{2 d /(d-2)}(\Omega)$ if $d>2$ ) and (ii) $\left\{\widetilde{\nabla}_{\mathcal{D}_{n}} u_{n}\right\}_{n \in \mathbb{N}}$ weakly converges to $\nabla \widetilde{u}$ in $\left[L^{2}(\Omega)\right]^{d}$. It only remains to prove that $\widetilde{u}=u$. Let $\varphi \in \mathfrak{D}$. Owing to (2.7) together with (P2),

$$
\left\|\widetilde{\nabla}_{\mathcal{D}_{n}}\left(u_{n}-\varphi_{\mathcal{T}_{n}}\right)\right\|_{\left[L^{2}(\Omega)\right]^{d}}^{2} \leq d\left\|u_{n}-\varphi_{\mathcal{T}_{n}}\right\|_{\mathcal{T}_{n}, I}^{2} \leq \frac{d}{\gamma_{1}} a_{\mathcal{T}_{n}}\left(u_{n}-\varphi_{\mathcal{T}_{n}}, u_{n}-\varphi_{\mathcal{T}_{n}}\right)=\frac{d}{\gamma_{1}}\left(T_{1}+T_{2}\right),
$$

where $T_{1} \stackrel{\text { def }}{=} \int_{\Omega} f(x)\left(u_{n}-\varphi_{\mathcal{T}_{n}}\right)(x) \mathrm{d} x$ and $T_{2} \stackrel{\text { def }}{=} a_{\mathcal{T}_{n}}\left(\varphi_{\mathcal{T}_{n}}, \varphi_{\mathcal{T}_{n}}-u_{n}\right)$. Clearly, by the integrability assumptions on $f$ and the weak convergence of $\left\{u_{n}\right\}_{n \in \mathbb{N}}$ to $\widetilde{u}$ in $L^{q}(\Omega)$ for all $q<+\infty$ if $d=2$ and for $q=\frac{2 d}{d-2}$ if $d>2$,

$$
T_{1} \rightarrow \int_{\Omega} f(x)(\widetilde{u}-\varphi)(x) \mathrm{d} x \text { as } n \rightarrow \infty
$$

Owing to $(2.10)$,

$$
\begin{aligned}
a_{\mathcal{T}_{n}}\left(\varphi_{\mathcal{T}_{n}}, u_{n}\right)= & \sum_{K \in \mathcal{T}_{n}} \sum_{\sigma \in \mathcal{E}_{K}}\left[\sqrt{\frac{d_{K, \sigma}}{\mathrm{m}_{\sigma}}} F_{K, \sigma}\left(\varphi_{\mathcal{T}_{n}}\right)-\mathrm{m}_{\sigma}\langle\Lambda \nabla \varphi\rangle_{K} \cdot \mathbf{n}_{K, \sigma}\right] \sqrt{\frac{\mathrm{m}_{\sigma}}{d_{K, \sigma}}}\left(\gamma_{\sigma} u_{n}-u_{n, K}\right) \\
& +\int_{\Omega} \Lambda(x) \nabla \varphi(x) \cdot \widetilde{\nabla}_{\mathcal{D}_{n}} u_{n}(x) \mathrm{d} x \stackrel{\text { def }}{=} T_{2,1}+T_{2,2} .
\end{aligned}
$$

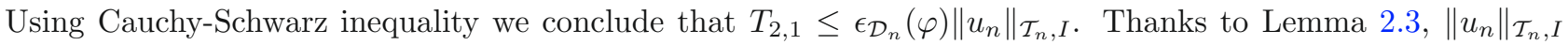
is bounded uniformly with respect to $n$. Thus, by property (P3), $T_{2,1} \rightarrow 0$ as $n \rightarrow \infty$. Also, using the weak convergence of $\left\{\widetilde{\nabla}_{\mathcal{D}_{n}} u_{n}\right\}_{n \in \mathbb{N}}$, we conclude that $T_{2,2} \rightarrow \int_{\Omega} \Lambda(x) \nabla \varphi(x) \cdot \nabla \widetilde{u}(x) \mathrm{d} x$ as $n \rightarrow \infty$. Let us now consider the remaining terms in $T_{2}$. By Proposition 2.1, $\left\|\varphi_{\mathcal{T}_{n}}\right\|_{\mathcal{T}_{n}, I}$ is uniformly bounded with respect to $n$; since $\varphi_{\mathcal{T}_{n}}$ obviously converges to $\varphi$, it is then easy, using Lemma 2.2 , to see that $\widetilde{\nabla}_{\mathcal{D}_{n}} \varphi_{\mathcal{T}_{n}}$ weakly converges to $\nabla \varphi$. Proceeding in a similar way as for $a_{\mathcal{T}_{n}}\left(\varphi_{\mathcal{T}_{n}}, u_{n}\right)$, we can thus prove that $a_{\mathcal{T}_{n}}\left(\varphi_{\mathcal{T}_{n}}, \varphi_{\mathcal{T}_{n}}\right) \rightarrow \int_{\Omega} \Lambda(x) \nabla \varphi(x) \cdot \nabla \varphi(x) \mathrm{d} x$ as $n \rightarrow \infty$. Therefore,

$$
T_{2} \rightarrow \int_{\Omega} \Lambda(x) \nabla \varphi(x) \cdot \nabla(\varphi-\widetilde{u})(x) \mathrm{d} x \text { as } n \rightarrow \infty .
$$

Plugging (2.13) and (2.14) into the right hand side of (2.12) and using the weak convergence of $\widetilde{\nabla}_{\mathcal{D}_{n}}\left(u_{n}-\varphi_{\mathcal{T}_{n}}\right)$, we conclude that, for all $\varphi \in \mathfrak{D}$,

$$
\|\nabla(\widetilde{u}-\varphi)\|_{\left[L^{2}(\Omega)\right]^{d}}^{2} \leq \frac{d}{\gamma_{1}}\left(\int_{\Omega} f(x)(\widetilde{u}-\varphi)(x) \mathrm{d} x+\int_{\Omega} \Lambda(x) \nabla \varphi(x) \cdot \nabla(\varphi-\widetilde{u})(x) \mathrm{d} x\right) .
$$

By virtue of (P1), we can apply this inequality to a sequence $\left\{\varphi_{m}\right\}_{m \in \mathbb{N}} \in \mathfrak{D}$ which tends to $\bar{u}$ in $H_{0}^{1}(\Omega)$ and let $m \rightarrow \infty$; since $\bar{u}$ solves problem (2.1), we obtain

$$
\|\nabla(\widetilde{u}-\bar{u})\|_{\left[L^{2}(\Omega)\right]^{d}}^{2} \leq \frac{d}{\gamma_{1}}\left[\int_{\Omega} f(x)(\widetilde{u}(x)-\bar{u}(x)) \mathrm{d} x-\int_{\Omega} \Lambda(x) \nabla \bar{u}(x) \cdot \nabla(\widetilde{u}-\bar{u})(x) \mathrm{d} x\right]=0,
$$

i.e., $\widetilde{u}=\bar{u}$. Due to the uniqueness of the solution of (2.1), we deduce that the entire sequence $\left\{u_{n}\right\}_{n \in \mathbb{N}}$ converges to $\bar{u}$ in $L^{q}(\Omega)$ for all $q \in\left[1,2 d /(d-2)\right.$ ) (and weakly in $L^{2 d /(d-2)}(\Omega)$ if $d>2$ ). Observe that the order in which the limits for $n \rightarrow \infty$ and $m \rightarrow \infty$ are taken cannot be exchanged, the sequence $\left\{\left\|\left(\varphi_{m}\right)_{\mathcal{T}_{n}}\right\|_{\mathcal{T}_{n}, I}\right\}_{m \in \mathbb{N}}$ being possibly unbounded. This concludes the proof. 


\subsection{A strongly convergent gradient reconstruction}

In practical applications, it is often desirable to dispose of a gradient reconstruction which strongly converges to the gradient of the continuous solution (whereas $\widetilde{\nabla}_{\mathcal{D}}$ only provides a weakly convergent approximation of this gradient). Such a reconstruction can be obtained using the same formula as in the Mixed Finite Volume method of Droniou and Eymard [19].

Let $\sigma \in \mathcal{E}$ be a mesh face, and denote by $x_{\sigma}$ its barycenter. For all $v \in H_{\mathcal{T}}(\Omega)$, define $\bar{\nabla}_{\mathcal{D}} v \in\left[H_{\mathcal{T}}(\Omega)\right]^{d}$ s.t., for all $K \in \mathcal{T}$,

$$
\bar{\nabla}_{\mathcal{D}} v(x)_{\mid K}=\frac{1}{\mathrm{~m}_{K}} \Lambda_{K}^{-1} \sum_{\sigma \in \mathcal{E}_{K}} F_{K, \sigma}(v)\left(x_{\sigma}-x_{K}\right) .
$$

For all $v \in H_{\mathcal{T}}$ and for all $K \in \mathcal{T},\left(\bar{\nabla}_{\mathcal{D}} v\right)_{K}$ will denote the (constant) value of $\bar{\nabla}_{\mathcal{D}} v$ on $K$, i.e., $\bar{\nabla}_{\mathcal{D}} v_{\mid K}(x)=$ $\left(\bar{\nabla}_{\mathcal{D}} v\right)_{K}$ for all $x \in K$. The following geometrical relation holds:

$$
\forall \xi \in \mathbb{R}^{d}, \forall K \in \mathcal{T}, \quad \sum_{\sigma \in \mathcal{E}_{K}} \mathrm{~m}_{\sigma} \xi \cdot \mathbf{n}_{K, \sigma}\left(x_{\sigma}-x_{K}\right)=\mathrm{m}_{K} \xi .
$$

Lemma 2.4 (consistency). Let $\left\{\mathcal{D}_{n}\right\}_{n \in \mathbb{N}}$ denote a family of discretizations matching Assumption 2.1 and s.t. $h_{\mathcal{D}_{n}} \rightarrow 0$ as $n \rightarrow \infty$. Then, for all $\varphi \in \mathfrak{D}$,

$$
\lim _{n \rightarrow \infty}\left\|\bar{\nabla}_{\mathcal{D}_{n}} \varphi_{\mathcal{T}_{n}}-\nabla \varphi\right\|_{\left[L^{2}(\Omega)\right]^{d}}^{2}=0
$$

Proof. For all $n \in \mathbb{N}$, for all $K \in \mathcal{T}_{n}$, formula (2.16) applied to $\xi=\langle\Lambda \nabla \varphi\rangle_{K}$ yields, for all $K \in \mathcal{T}$,

$$
\Lambda_{K}^{-1}\langle\Lambda \nabla \varphi\rangle_{K}=\frac{1}{\mathrm{~m}_{K}} \Lambda_{K}^{-1} \sum_{\sigma \in \mathcal{E}_{K}} \mathrm{~m}_{\sigma}\langle\Lambda \nabla \varphi\rangle_{K} \cdot \mathbf{n}_{K, \sigma}\left(x_{\sigma}-x_{K}\right) .
$$

Let $T_{K, \sigma}\left(\varphi_{\mathcal{T}}\right) \stackrel{\text { def }}{=} F_{K, \sigma}\left(\varphi_{\mathcal{T}}\right)-\mathrm{m}_{\sigma}\langle\Lambda \nabla \varphi\rangle_{K} \cdot \mathbf{n}_{K, \sigma}$. Using Cauchy-Schwarz inequality we get, for all $K \in \mathcal{T}$ and for all $y \in K$,

$$
\begin{aligned}
\left|\left(\bar{\nabla}_{\mathcal{D}_{n}} \varphi_{\mathcal{T}_{n}}\right)_{K}-\nabla \varphi(y)\right| & =\left|\frac{1}{\mathrm{~m}_{K}} \Lambda_{K}^{-1} \sum_{\sigma \in \mathcal{E}_{K}} T_{K, \sigma}\left(\varphi_{\mathcal{T}}\right)\left(x_{\sigma}-x_{K}\right)+\frac{1}{\mathrm{~m}_{K}} \Lambda_{K}^{-1} \int_{K} \Lambda(x)(\nabla \varphi(x)-\nabla \varphi(y)) \mathrm{d} x\right| \\
& \leq \frac{1}{\alpha_{0} \mathrm{~m}_{K}}\left[\sum_{\sigma \in \mathcal{E}_{K}} \sqrt{\frac{d_{K, \sigma}}{\mathrm{m}_{\sigma}}}\left|T_{K, \sigma}\left(\varphi_{\mathcal{T}_{n}}\right)\right| \frac{\left|x_{\sigma}-x_{K}\right|}{\sqrt{d_{K, \sigma}}} \sqrt{\mathrm{m}_{\sigma}}+C_{3} \beta_{0} \mathrm{~m}_{K} \operatorname{diam}(K)\right] \\
& \leq \frac{1}{\alpha_{0} \mathrm{~m}_{K}}\left(\sum_{\sigma \in \mathcal{E}_{K}} \frac{d_{K, \sigma}}{\mathrm{m}_{\sigma}}\left|T_{K, \sigma}\left(\varphi_{\mathcal{T}_{n}}\right)\right|^{2}\right)^{\frac{1}{2}}\left(\sum_{\sigma \in \mathcal{E}_{K}} \frac{\left|x_{\sigma}-x_{K}\right|^{2}}{d_{K, \sigma}} \mathrm{m}_{\sigma}\right)^{\frac{1}{2}}+\frac{C_{3} \beta_{0}}{\alpha_{0}} \operatorname{diam}(K)
\end{aligned}
$$

where $C_{3} \stackrel{\text { def }}{=} \sup _{x \in \Omega_{i}, i=1 \ldots N_{\Omega}}\left|\varphi^{\prime \prime}(x)\right|$. As a consequence, using (2.2) together with (2.3),

$$
\begin{aligned}
\int_{K}\left|\left(\bar{\nabla}_{\mathcal{D}_{n}} \varphi_{\mathcal{T}_{n}}\right)_{K}-\nabla \varphi(y)\right|^{2} \mathrm{~d} y \leq & \frac{2}{\left(\alpha_{0} \varrho_{1}\right)^{2}} \sum_{\sigma \in \mathcal{E}_{K}} \frac{d_{K, \sigma}}{\mathrm{m}_{\sigma}}\left|T_{K, \sigma}\left(\varphi_{\mathcal{T}_{n}}\right)\right|^{2} \times \frac{1}{\mathrm{~m}_{K}} \sum_{\sigma \in \mathcal{E}_{K}} \mathrm{~m}_{\sigma} d_{K, \sigma} \\
& +2\left(\frac{C_{3} \beta_{0}}{\alpha_{0}}\right)^{2} \mathrm{~m}_{K} \operatorname{diam}(K)^{2} \\
\leq & \frac{2 d}{\left(\alpha_{0} \varrho_{1}\right)^{2}} \sum_{\sigma \in \mathcal{E}_{K}} \frac{d_{K, \sigma}}{\mathrm{m}_{\sigma}}\left|T_{K, \sigma}\left(\varphi_{\mathcal{T}_{n}}\right)\right|^{2}+2\left(\frac{C_{3} \beta_{0}}{\alpha_{0}}\right)^{2} \mathrm{~m}_{K} h_{\mathcal{D}_{n}}^{2} .
\end{aligned}
$$


Finally, summing over $K \in \mathcal{T}_{n}$,

$$
\left\|\bar{\nabla}_{\mathcal{D}_{n}} \varphi_{\mathcal{T}_{n}}-\nabla \varphi\right\|_{\left[L^{2}(\Omega)\right]^{d}}^{2} \leq \frac{2 d}{\left(\alpha_{0} \varrho_{1}\right)^{2}} \epsilon_{\mathcal{D}_{n}}^{2}(\varphi)+2\left(\frac{C_{3} \beta_{0}}{\alpha_{0}}\right)^{2} \mathrm{~m}_{\Omega} h_{\mathcal{D}_{n}}^{2}
$$

Use (P3) to conclude.

The convergence of the gradient reconstruction (2.15) requires the following:

Assumption 2.2. Assume that there is $C_{4}>0$ s.t.

$$
\forall n \in \mathbb{N}, \forall v \in H_{\mathcal{T}_{n}}(\Omega), \quad \sum_{K \in \mathcal{T}_{n}} \sum_{\sigma \in \mathcal{E}_{K}} \frac{d_{K, \sigma}}{\mathrm{m}_{\sigma}}\left|F_{K, \sigma}(v)\right|^{2} \leq C_{4}\|v\|_{\mathcal{T}_{n}, I}^{2}
$$

Remark 2.5. Assumption 2.2 is readily verified on $\Lambda$-orthogonal meshes and two point fluxes. A mesh is said to be $\Lambda$-orthogonal if, for all $\sigma \in \mathcal{E}$, there exists $x_{\sigma} \in \sigma$ s.t., for all $K \in \mathcal{T}_{\sigma}, \Lambda_{K}^{-1}\left(x_{\sigma}-x_{K}\right)$ is orthogonal to $\sigma$. For $\Lambda$-orthogonal meshes, we can define two-points consistent fluxes in the sense of $(2.9)$ as $F_{K, \sigma}(v)=\mathrm{m}_{\sigma} t_{\sigma} \frac{\left(\gamma_{\sigma} v-v_{K}\right)}{d_{K, \sigma}}$, where the reals $\left\{t_{\sigma}\right\}_{\sigma \in \mathcal{E}}$ are given by

$$
\begin{cases}\frac{d_{K, \sigma}}{\Lambda_{K} \mathbf{n}_{K, \sigma} \cdot \mathbf{n}_{K, \sigma}}+\frac{d_{L, \sigma}}{\Lambda_{L} \mathbf{n}_{L, \sigma} \cdot \mathbf{n}_{L, \sigma}}=\frac{d_{K, \sigma}+d_{L, \sigma}}{t_{\sigma}} & \text { if } \sigma \in \mathcal{E}_{\text {int }} \text { with } \mathcal{T}_{\sigma}=\{K, L\} \\ t_{\sigma}=\Lambda_{K} \mathbf{n}_{K, \sigma} \cdot \mathbf{n}_{K, \sigma} & \text { if } \sigma \in \mathcal{E}_{\text {ext }} \text { with } \mathcal{T}_{\sigma}=\{K\} .\end{cases}
$$

Since for all $\sigma \in \mathcal{E}, t_{\sigma} \leq \beta_{0}$, (2.17) holds with $C_{4}=\beta_{0}^{2}$. Inequality (2.17) can be interpreted as a stability requirement on the numerical fluxes. In particular, for the G-method of Section 3, Assumption 2.2 is proved for general meshes in Lemma 3.5 below.

Proposition 2.2 (stability of the gradient reconstruction). Let $\left\{\mathcal{D}_{n}\right\}_{n \in \mathbb{N}}$ denote a family of discretizations matching Definition 2.1. Let Assumption 2.2 hold. Then,

$$
\forall v \in H_{\mathcal{T}}(\Omega), \quad\left\|\bar{\nabla}_{\mathcal{D}} v\right\|_{\left[L^{2}(\Omega)\right]^{d}} \leq \frac{\sqrt{d C_{4}}}{\alpha_{0} \varrho_{1}}\|v\|_{\mathcal{T}, I}
$$

Proof. Let $v \in H_{\mathcal{T}}(\Omega)$. Cauchy-Schwarz inequality yields

$$
\begin{aligned}
\left\|\bar{\nabla}_{\mathcal{D}} v\right\|_{\left[L^{2}(\Omega)\right]^{d}}^{2} & =\sum_{K \in \mathcal{T}} \mathrm{m}_{K}\left|\frac{1}{\mathrm{~m}_{K}} \Lambda_{K}^{-1} \sum_{\sigma \in \mathcal{E}_{K}} F_{K, \sigma}(v)\left(x_{\sigma}-x_{K}\right)\right|^{2} \\
& \leq \frac{1}{\alpha_{0}^{2}} \sum_{K \in \mathcal{T}}\left(\sum_{\sigma \in \mathcal{E}_{K}} \frac{d_{K, \sigma}}{\mathrm{m}_{\sigma}}\left|F_{K, \sigma}(v)\right|^{2} \times \sum_{\sigma \in \mathcal{E}_{K}} \frac{\mathrm{m}_{\sigma} \operatorname{diam}(K)^{2}}{d_{K, \sigma} \mathrm{m}_{K}}\right) .
\end{aligned}
$$

Owing to (2.3) together with (2.2), $\sum_{\sigma \in \mathcal{E}_{K}} \frac{\mathrm{m}_{\sigma} \operatorname{diam}(K)^{2}}{d_{K, \sigma} \mathrm{m}_{K}} \leq \frac{d}{\varrho_{1}^{2}}$. Conclude using Assumption 2.2.

Theorem 2.2 (strong convergence of the discrete gradient reconstruction). Let $\bar{u}$ be the solution to (2.1). Let $\left\{\mathcal{D}_{n}\right\}_{n \in \mathbb{N}}$ be a family of meshes matching Definition 2.1 and s.t. $h_{\mathcal{D}_{n}} \rightarrow 0$ as $n \rightarrow \infty$, and denote by $u_{n}$ the solution of problem (2.5) on $\mathcal{D}_{n}$. Finally, let Assumptions 2.1 and 2.2 hold. Then, the sequence $\left\{\bar{\nabla}_{\mathcal{D}_{n}} u_{n}\right\}_{n \in \mathbb{N}}$ strongly converges to $\nabla \bar{u}$ in $\left[L^{2}(\Omega)\right]^{d}$.

Proof. Thanks to Theorem 2.1 together with Lemma 2.2, (i) the sequence of weak solutions $\left\{u_{n}\right\}_{n \in \mathbb{N}}$ converges to $\bar{u}$ in $L^{q}(\Omega)$, for all $q \in[1,2 d /(d-2))$ and (ii) the sequence $\left\{\widetilde{\nabla}_{\mathcal{D}_{n}} u_{n}\right\}_{n \in \mathbb{N}}$ weakly converges to $\nabla \bar{u}$ in $\left[L^{2}(\Omega)\right]^{d}$. 
Let $\varphi \in \mathfrak{D}$. For all $n \in \mathbb{N}$,

$$
\int_{\Omega}\left|\bar{\nabla}_{\mathcal{D}_{n}} u_{n}-\nabla \bar{u}\right|^{2} \leq 3\left(\int_{\Omega}\left|\bar{\nabla}_{\mathcal{D}_{n}} u_{n}-\bar{\nabla}_{\mathcal{D}_{n}} \varphi_{\mathcal{T}_{n}}\right|^{2}+\int_{\Omega}\left|\bar{\nabla}_{\mathcal{D}_{n}} \varphi_{\mathcal{T}_{n}}-\nabla \varphi\right|^{2}+\int_{\Omega}|\nabla \varphi(x)-\nabla \bar{u}|^{2}\right) .
$$

Let $T_{i}, i=1 \ldots 3$ denote the addends in the right hand side. Owing to Proposition 2.2 together with (P2) we have

$$
T_{1} \leq \frac{d C_{4}}{\left(\alpha_{0} \varrho_{1}\right)^{2} \gamma_{1}} a_{\mathcal{T}_{n}}\left(u_{n}-\varphi_{\mathcal{T}_{n}}, u_{n}-\varphi_{\mathcal{T}_{n}}\right) .
$$

Using the same arguments as in the proof of Theorem 2.1, we infer that

$$
\limsup _{n \rightarrow \infty} T_{1} \leq \frac{d C_{4}}{\left(\alpha_{0} \varrho_{1}\right)^{2} \gamma_{1}}\left(\int_{\Omega} f(\bar{u}-\varphi)+\int_{\Omega} \Lambda \nabla \varphi \cdot(\nabla \varphi-\nabla \bar{u})\right) .
$$

Moreover, owing to Lemma $2.4, \lim _{n \rightarrow \infty} T_{2}=0$ and thus

$$
\limsup _{n \rightarrow \infty} \int_{\Omega}\left|\bar{\nabla}_{\mathcal{D}_{n}} u-\nabla \bar{u}\right|^{2} \leq 3 \frac{d C_{4}}{\left(\alpha_{0} \varrho_{1}\right)^{2} \gamma_{1}}\left(\int_{\Omega} f(\bar{u}-\varphi)+\int_{\Omega} \Lambda \nabla \varphi \cdot(\nabla \varphi-\nabla \bar{u})\right)+3\left(\int_{\Omega}|\nabla \varphi-\nabla \bar{u}|^{2}\right) .
$$

Finally, since $\mathfrak{D}$ is dense in $H_{0}^{1}(\Omega)$, we conclude by letting $\varphi$ tend to $\bar{u}$ in $H_{0}^{1}(\Omega)$ as in the proof of Theorem 2.1.

\section{The G MEthod}

In this section we introduce a family of FV methods generalizing the MPFA L method of $[3,5]$ and show that it fits in the abstract analysis framework of Section 2.

\subsection{Construction of group gradients}

The following definition collects the additional requirements on the discretization family $\left\{\mathcal{D}_{n}\right\}_{n \in \mathbb{N}}$ for the G-method to be applicable.

Definition 3.1. Let $\left\{\mathcal{D}_{n}\right\}_{n \in \mathbb{N}}$ be a family of meshes matching Definition 2.1. We further suppose that:

(i) $\mathcal{V}_{n}$ is a family of points (the vertices or nodes), s.t., for all $K \in \mathcal{T}_{n}$, for all $U_{K} \subseteq \mathcal{E}_{K}$ satisfying $\operatorname{card}\left(U_{K}\right) \geq d$, we have $\bigcap_{\sigma \in U_{K}} \sigma=\emptyset$ or $\bigcap_{\sigma \in U_{K}} \sigma=s \in \mathcal{V}_{n}$. For all $s \in \mathcal{V}_{n}$, we let $\mathcal{E}_{s} \stackrel{\text { def }}{=}\{\sigma \in \mathcal{E} \mid s \in \sigma\}$ and $\mathcal{T}_{s} \stackrel{\text { def }}{=}\{K \in \mathcal{T} \mid s \in \bar{K}\}$, and we assume that each $\sigma$ contains at least one vertex (this could be false in dimension $d=3$ if, for example, $\sigma$ is only a portion of a planar face of a cell);

(ii) the number of faces sharing one node remains bounded as the mesh is refined, i.e., there exists $\varrho_{3}$ s.t.

$$
\sup _{n \in \mathbb{N}} \max _{s \in \mathcal{V}_{n}} \operatorname{card}\left(\mathcal{E}_{s}\right) \leq \varrho_{3} ;
$$

(iii) $\widetilde{\mathcal{G}}$ is the finite family of face groups defined as follows:

$$
\widetilde{\mathcal{G}} \stackrel{\text { def }}{=}\left\{G \subset \mathcal{E}_{K} \cap \mathcal{E}_{s}, K \in \mathcal{T}_{s}, s \in \mathcal{V}_{n}, \operatorname{card}(G)=d\right\} .
$$

For each $G \in \widetilde{\mathcal{G}}$, we let $\mathcal{T}_{G} \stackrel{\text { def }}{=}\left\{K \in \mathcal{T} \mid G \cap \mathcal{E}_{K} \neq \emptyset\right\}$. We also arbitrarily select a cell, which we denote by $K_{G}$, s.t. $G \subset \mathcal{E}_{K_{G}}$ (see Fig. 2).

Remark 3.1. In many cases, a given group $G$ is contained in a unique $\mathcal{E}_{K}$ but, in some cases (especially if the discretization has non-convex cells), there can be multiple possible choices for $K_{G}$. In [3,5] the cell $K_{G}$ is referred to as the primary cell. 

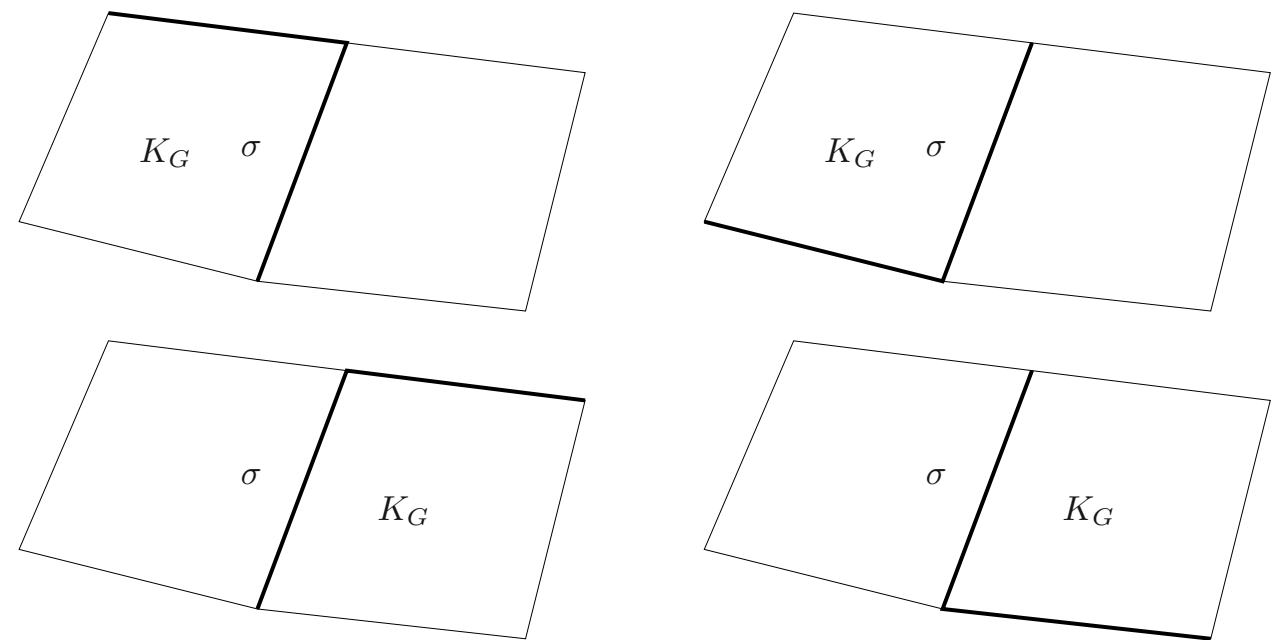

Figure 2. Groups containing a face $\sigma$ and corresponding primary cells $K_{G}$ for $d=2$.

Let $v \in H_{\mathcal{T}}(\Omega), G \in \widetilde{\mathcal{G}}, \sigma \in G$ and $K \in \mathcal{T}_{\sigma}$. In order to define a discrete flux $F_{K, \sigma}(v)$ through $\sigma$, we construct a group gradient $\left(\nabla_{\mathcal{D}} v\right)_{K}^{G, \sigma} \in \mathbb{R}^{d}$. The full gradient used in the definition of $F_{K, \sigma}(v)$ will then be obtained as a convex combination of the group gradients corresponding to the groups $G \in \mathcal{G}$ s.t. $\sigma \in G$ (see Eq. (3.6) below). Let us detail the procedure to obtain the group gradient $\left(\nabla_{\mathcal{D}} v\right)_{K}^{G, \sigma}$. First, for all $\sigma \in \mathcal{E}, \mathcal{T}_{\sigma}=\{K, L\}$, we require that the values $v_{K}, v_{L}$ and the gradient reconstruction $\left(\nabla_{\mathcal{D}} v\right)_{K}^{G, \sigma},\left(\nabla_{\mathcal{D}} v\right)_{L}^{G, \sigma}$ yield the same value trace on $\sigma$, i.e.,

$$
v_{K}+\left(\nabla_{\mathcal{D}} v\right)_{K}^{G, \sigma} \cdot\left(x-x_{K}\right)=v_{L}+\left(\nabla_{\mathcal{D}} v\right)_{L}^{G, \sigma} \cdot\left(x-x_{L}\right) \quad \forall x \in \sigma
$$

For a boundary face $\sigma$, we require that the value obtained at its barycenter $x_{\sigma}$ be zero. Second, we require the conservativity of the resulting fluxes, i.e.,

$$
\Lambda_{K}\left(\nabla_{\mathcal{D}} v\right)_{K}^{G, \sigma} \cdot \mathbf{n}_{K, \sigma}+\Lambda_{L}\left(\nabla_{\mathcal{D}} v\right)_{L}^{G, \sigma} \cdot \mathbf{n}_{L, \sigma}=0
$$

The above set of equations are not sufficient to uniquely define the group gradients (and thus to estimate them, which is fundamental in the study of the numerical method). We therefore add another constraint, giving a particular role to the cell $K_{G}$ selected for the group $G$, namely we require that $\left(\nabla_{\mathcal{D}} v\right)_{K_{G}}^{G, \sigma}$ do not depend on $\sigma \in G$, and we denote by $\left(\nabla_{\mathcal{D}} v\right)_{K_{G}}^{G}$ the common value of this group gradient for all $\sigma \in G$. The discrete gradients are thus defined, as the solution of the following local problems: For all $G \in \widetilde{\mathcal{G}}$ and all $\sigma \in G \cap \mathcal{E}_{\text {int }}$, with $\mathcal{T}_{\sigma}=\left\{K_{G}, L\right\}$

$$
\left\{\begin{array}{l}
v_{K_{G}}+\left(\nabla_{\mathcal{D}} v\right)_{K_{G}}^{G} \cdot\left(x-x_{K_{G}}\right)=v_{L}+\left(\nabla_{\mathcal{D}} v\right)_{L}^{G, \sigma} \cdot\left(x-x_{L}\right) \quad \forall x \in \sigma, \\
\Lambda_{K_{G}}\left(\nabla_{\mathcal{D}} v\right)_{K_{G}}^{G} \cdot \mathbf{n}_{K_{G}, \sigma}+\Lambda_{L}\left(\nabla_{\mathcal{D}} v\right)_{L}^{G, \sigma} \cdot \mathbf{n}_{L, \sigma}=0
\end{array}\right.
$$

and for all $\sigma \in G \cap \mathcal{E}_{\text {ext }}$,

$$
v_{K_{G}}+\left(\nabla_{\mathcal{D}} v\right)_{K_{G}}^{G} \cdot\left(x_{\sigma}-x_{K_{G}}\right)=0 .
$$

Lemma 3.1. For each group $G \in \mathcal{G}$, the gradient reconstruction $\left(\nabla_{\mathcal{D}} v\right)_{K_{G}}^{G}$ defined by (3.1) and (3.2) can be obtained solving a linear system of the form

$$
\mathcal{A}_{G} X_{G}=\mathcal{B}_{G}(v)
$$


where $\mathcal{A}_{G} \in \mathbb{R}^{d, d}$ is defined row-wise by

$$
\mathcal{A}_{G}=\left(\begin{array}{c}
\left(\frac{\Lambda_{L} \mathbf{n}_{L, \sigma} \cdot \mathbf{n}_{L, \sigma}}{d_{L, \sigma}}\left(x_{L}-x_{K_{G}}\right)+\Lambda_{K_{G}} \mathbf{n}_{K_{G}, \sigma}+\Lambda_{L} \mathbf{n}_{L, \sigma}\right)_{\sigma \in G \cap \mathcal{E}_{\mathrm{int}}}^{t} \\
\left(\frac{\Lambda_{K_{G}} \mathbf{n}_{K_{G}, \sigma} \cdot \mathbf{n}_{K_{G}, \sigma}}{d_{K_{G}, \sigma}}\left(x_{\sigma}-x_{K_{G}}\right)\right)_{\sigma \in G \cap \mathcal{E}_{\mathrm{ext}}}^{t}
\end{array}\right),
$$

and $\mathcal{B}_{G}(v) \in \mathbb{R}^{d}$ is defined as

$$
\mathcal{B}_{G}(v)=\left(\begin{array}{l}
\left(\frac{\Lambda_{L} \mathbf{n}_{L, \sigma} \cdot \mathbf{n}_{L, \sigma}}{d_{L, \sigma}}\left(v_{L}-v_{K_{G}}\right)\right)_{\sigma \in G \cap \mathcal{E}_{\mathrm{int}}} \\
\left(\frac{\Lambda_{K_{G}} \mathbf{n}_{K_{G}, \sigma} \cdot \mathbf{n}_{K_{G}, \sigma}}{d_{K_{G}, \sigma}}\left(-v_{K_{G}}\right)\right)_{\sigma \in G \cap \mathcal{E}_{\mathrm{ext}}}
\end{array}\right) .
$$

Proof. Let $v \in H_{\mathcal{T}}(\Omega), G \in \mathcal{G}, \sigma \in G \cap \mathcal{E}_{\text {int }}$ with $\mathcal{T}_{\sigma}=\left\{K_{G}, L\right\}$. Observe that, if $\mathbf{v} \stackrel{\text { def }}{=}\left(\nabla_{\mathcal{D}} v\right)_{K_{G}}^{G}-\left(\nabla_{\mathcal{D}} v\right)_{L}^{G, \sigma} \neq 0$, the first equation of (3.1) is the equation of an hyperplane of $\mathbb{R}^{d}$ orthogonal to $\mathbf{v}$; satisfying this equation for all $x \in \sigma$ is equivalent to imposing that $\sigma$ is contained in this hyperplane, and thus that $\mathbf{v}$ and $\mathbf{n}_{K_{G}, \sigma}$ are colinear (this is of course also true if $\mathbf{v}=0$ ). As a consequence, taking $y_{\sigma} \in \sigma$, the first equation in (3.1) is equivalent to the following linear system (in which $\lambda_{\sigma}^{G} \in \mathbb{R}$ is an additional unknown):

$$
\left\{\begin{array}{l}
\left(\nabla_{\mathcal{D}} v\right)_{K_{G}}^{G}-\left(\nabla_{\mathcal{D}} v\right)_{L}^{G, \sigma}=\lambda_{\sigma}^{G} \mathbf{n}_{K_{G}, \sigma} \\
v_{K_{G}}-v_{L}+\left(\nabla_{\mathcal{D}} v\right)_{L}^{G, \sigma} \cdot x_{L}-\left(\nabla_{\mathcal{D}} v\right)_{K_{G}}^{G} \cdot x_{K_{G}}=-\lambda_{\sigma}^{G} \mathbf{n}_{K_{G}, \sigma} \cdot y_{\sigma} .
\end{array}\right.
$$

Since, by point (iii) in Definition 2.1, $\left(y_{\sigma}-x_{L}\right) \cdot \mathbf{n}_{K_{G}, \sigma}=-d_{L, \sigma}>0$, solving for $\lambda_{\sigma}^{G}$ we obtain

$$
\left\{\begin{array}{l}
\lambda_{\sigma}^{G}=-\frac{R_{L, \sigma}(v)}{d_{L, \sigma}} \\
\left(\nabla_{\mathcal{D}} v\right)_{L}^{G, \sigma}=\left(\nabla_{\mathcal{D}} v\right)_{K_{G}}^{G}-\frac{R_{L, \sigma}(v)}{d_{L, \sigma}} \mathbf{n}_{L, \sigma}
\end{array}\right.
$$

with $R_{L, \sigma}(v) \stackrel{\text { def }}{=} v_{L}-v_{K_{G}}-\left(\nabla_{\mathcal{D}} v\right)_{K_{G}}^{G} \cdot\left(x_{L}-x_{K_{G}}\right)$. Using these expressions, the second equation of (3.1) can be rewritten as

$$
\left[\Lambda_{K_{G}} \mathbf{n}_{K_{G}, \sigma}+\Lambda_{L} \mathbf{n}_{L, \sigma}+\frac{\Lambda_{L} \mathbf{n}_{L, \sigma} \cdot \mathbf{n}_{L, \sigma}}{d_{L, \sigma}}\left(x_{L}-x_{K_{G}}\right)\right] \cdot\left(\nabla_{\mathcal{D}} v\right)_{K_{G}}^{G}=\frac{\Lambda_{L} \mathbf{n}_{L, \sigma} \cdot \mathbf{n}_{L, \sigma}}{d_{L, \sigma}}\left(v_{L}-v_{K_{G}}\right)
$$

The linear system (3.1)-(3.2) is thus equivalent to:

$$
\left\{\begin{array}{c}
\left(\nabla_{\mathcal{D}} v\right)_{L}^{G, \sigma}=\left(\nabla_{\mathcal{D}} v\right)_{K_{G}}^{G}-\frac{R_{L, \sigma}(v)}{d_{L, \sigma}} \mathbf{n}_{L, \sigma}, \quad \forall \sigma \in G \cap \mathcal{E}_{\mathrm{int}}, \mathcal{T}_{\sigma}=\left\{K_{G}, L\right\}, \\
{\left[\frac{\Lambda_{L} \mathbf{n}_{L, \sigma} \cdot \mathbf{n}_{L, \sigma}}{d_{L, \sigma}}\left(x_{L}-x_{K_{G}}\right)+\Lambda_{K_{G}} \mathbf{n}_{K_{G}, \sigma}+\Lambda_{L} \mathbf{n}_{L, \sigma}\right] \cdot\left(\nabla_{\mathcal{D}} v\right)_{K_{G}}^{G}} \\
=\frac{\Lambda_{L} \mathbf{n}_{L, \sigma} \cdot \mathbf{n}_{L, \sigma}}{d_{L, \sigma}}\left(v_{L}-v_{K_{G}}\right), \quad \forall \sigma \in G \cap \mathcal{E}_{\mathrm{int}}, \mathcal{T}_{\sigma}=\left\{K_{G}, L\right\}, \\
\frac{\Lambda_{K_{G}} \mathbf{n}_{K_{G}, \sigma} \cdot \mathbf{n}_{K_{G}, \sigma}}{d_{K_{G}, \sigma}}\left(\nabla_{\mathcal{D}} v\right)_{K_{G}}^{G} \cdot\left(x_{\sigma}-x_{K_{G}}\right)=\frac{\Lambda_{K_{G}} \mathbf{n}_{K_{G}, \sigma} \cdot \mathbf{n}_{K_{G}, \sigma}}{d_{K_{G}, \sigma}}\left(-v_{K_{G}}\right), \quad \forall \sigma \in G \cap \mathcal{E}_{\mathrm{ext}} .
\end{array}\right.
$$

We introduce the set of groups s.t. the matrix $\mathcal{A}_{G}$ is invertible, that is to say

$$
\mathcal{G} \stackrel{\text { def }}{=}\left\{G \in \widetilde{\mathcal{G}} \mid \mathcal{A}_{G} \text { is invertible }\right\} .
$$




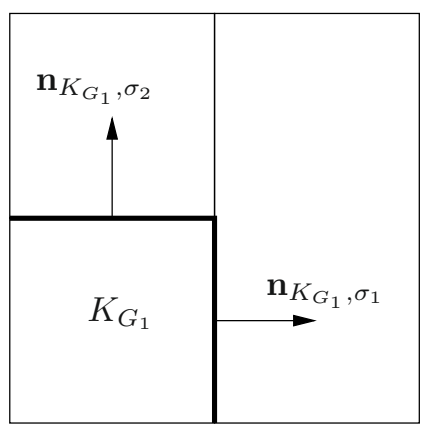

(a) $G_{1} \in \mathcal{G}_{\sigma}$

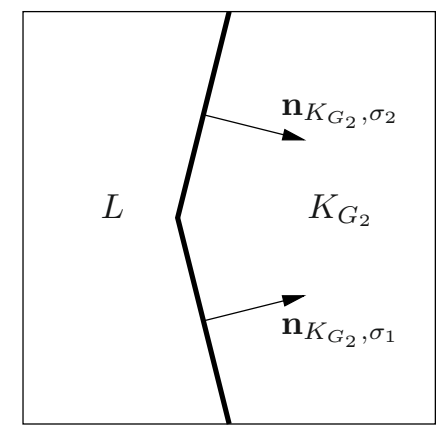

(b) $G_{2} \notin \mathcal{G}_{\sigma}$

Figure 3. Two examples of face groups of $\widetilde{\mathcal{G}}$ respectively belonging and not belonging to $\mathcal{G}_{\sigma}$.

We infer from the expression provided in Lemma 3.1 that the invertibility of the matrices $\left\{\mathcal{A}_{G}\right\}_{G \in \widetilde{\mathcal{G}}}$ depends on both the mesh and the permeability tensor. As a result, on a given mesh, the matrix associated to one group $G \in \widetilde{\mathcal{G}}$ may or may not be invertible depending on the values of $\Lambda$ on the cells of $\mathcal{T}_{G}$. For all $\sigma \in \mathcal{E}$, define the set of groups containing $\sigma$ as

$$
\mathcal{G}_{\sigma} \stackrel{\text { def }}{=}\{G \in \mathcal{G} \mid \sigma \in G\}
$$

In order to construct the fluxes $F_{K, \sigma}, K \in \mathcal{T}_{\sigma}$, at least one group $G \in \mathcal{G}$ containing $\sigma$ must be available. As a consequence, throughout the rest of this work, we shall assume that:

\section{Assumption 3.1. For all $\sigma \in \mathcal{E}, \mathcal{G}_{\sigma}$ is non-empty.}

In practical applications, Assumption 3.1 can be verified in a preliminary step. Should exist $\sigma \in \widetilde{\mathcal{G}}$ for which no group $G$ containing $\sigma$ yields an invertible matrix $\mathcal{A}_{G}$, recovery strategies can be devised. One possibility is, e.g., to proceed as in Eymard et al. [24] and locally hybridize the method by introducing a face unknown. Another possibility is to move the cell centers $\left\{x_{K}\right\}_{K \in \mathcal{T}_{\sigma}}$ still matching point (iii) in Definition 2.1. It has to be noticed, however, that this second strategy affects all faces $\sigma^{\prime} \in \mathcal{E}_{K}, K \in \mathcal{T}_{\sigma}$. In both cases, the convergence of the method can be proved following the abstract analysis framework of Section 2.

Figure 3 shows two examples of groups respectively belonging and not belonging to $\mathcal{G}$ (in the case where $\Lambda$ is constant). Indeed, since $\Lambda_{K_{G_{2}}}=\Lambda_{L}$, the terms $\Lambda_{K_{G_{2}}} \mathbf{n}_{K_{G_{2}}, \sigma_{i}}$ and $\Lambda_{L} \mathbf{n}_{L, \sigma_{i}}$ cancel out in each line of $\mathcal{A}_{G_{2}}$ and, since the cell $L$ on the other side of $\sigma_{1}$ and $\sigma_{2}$ is the same, both lines of $\mathcal{A}_{G_{2}}$ are colinear to $x_{L}-x_{K_{G_{2}}}$, and the matrix is singular. The non-convexity of cells can be a cause to the singularity of some $\mathcal{A}_{G}$ (but this does not block the use of the $\mathrm{G}$ method since, even in this case, the non-emptiness of all $\mathcal{G}_{\sigma}$ often holds).

Finally, we define in the following two lemmata the space playing the role of $\mathfrak{D}$ in Assumption 2.1, we state its density and we establish the consistency on this space of the group gradients (this will give (P3)).

Lemma 3.2 (density of a space of test-functions). Let $\mathcal{Q}$ be the space of functions $\varphi: \bar{\Omega} \rightarrow \mathbb{R}$ s.t.

(i) $\left(\varphi\right.$ is continuous and piecewise regular) $\varphi \in C_{0}(\bar{\Omega})$ and, for all $i=1, \ldots, N_{\Omega}, \varphi \in C^{2}\left(\overline{\Omega_{i}}\right)$;

(ii) (the tangential derivatives of $\varphi$ are continuous through the interfaces of $P_{\Omega}$ ) for all $i, j=1, \ldots, N_{\Omega}$, for all vector $\mathbf{t}$ parallel to $\partial \Omega_{i} \cap \partial \Omega_{j},(\nabla \varphi)_{\mid \overline{\Omega_{i}}} \cdot \mathbf{t}=(\nabla \varphi)_{\mid \overline{\Omega_{j}}} \cdot \mathbf{t}$, where $(\nabla \varphi)_{\mid \overline{\Omega_{i}}}$ refers to the value of $\nabla \varphi$ on $\partial \Omega_{i}$ computed from the values on $\overline{\Omega_{i}}$;

(iii) (the flux of $\nabla \varphi$ directed by $\Lambda \mathbf{n}$ is continuous through the interfaces of $P_{\Omega}$ ) for all $i, j=1, \ldots, N_{\Omega}$ s.t. $\partial \Omega_{i} \cap \partial \Omega_{j}$ has dimension $d-1,(\Lambda \nabla \varphi)_{\mid \overline{\Omega_{i}}} \cdot \mathbf{n}_{i}+(\Lambda \nabla \varphi)_{\mid \overline{\Omega_{j}}} \cdot \mathbf{n}_{j}=0$ on $\partial \Omega_{i} \cap \partial \Omega_{j}$, where $\mathbf{n}_{i}$ is the outer normal to $\Omega_{i}$.

Then, $\mathcal{Q}$ is dense in $H_{0}^{1}(\Omega)$.

Proof. See Appendix. 
Lemma 3.3 (consistency of the group gradients). Let $\mathcal{D}$ be an element of a family of discretizations satisfying Assumption 2.1. For all $\varphi \in \mathcal{Q}$, there exists a real $C_{5}>0$ which only depends on $\varrho_{1}, \varrho_{2}, \Lambda$ and $\varphi$ s.t., for all $G \in \mathcal{G}$, all $\sigma \in G$ and all $K \in \mathcal{T}_{\sigma}$,

$$
\left|\left(\nabla_{\mathcal{D}} \varphi_{\mathcal{T}}\right)_{K}^{G, \sigma}-\nabla \varphi\left(x_{K}\right)\right| \leq C_{5}\left(1+\left|\mathcal{A}_{G}^{-1}\right|\right) \max _{K \in \mathcal{T}_{G}} \operatorname{diam}(K) .
$$

Proof. See Appendix B.

\subsection{Numerical fluxes}

In this section we introduce the numerical fluxes computed from the group gradients of Section 3.1. We choose a set of weights $\left\{\theta_{\sigma}^{G}\right\}_{\sigma \in \mathcal{E}, G \in \mathcal{G}_{\sigma}}$ s.t.

$$
\text { For all } \sigma \in \mathcal{E} \text {, for all } G \in \mathcal{G}_{\sigma}, 0 \leq \theta_{\sigma}^{G} \leq 1 \text { and, for all } \sigma \in \mathcal{E}, \sum_{G \in \mathcal{G}_{\sigma}} \theta_{\sigma}^{G}=1 .
$$

The numerical fluxes are then defined as follows: For all $K \in \mathcal{T}$, for all $\sigma \in \mathcal{E}_{K}$,

$$
F_{K, \sigma}(u) \stackrel{\text { def }}{=} \sum_{G \in \mathcal{G}_{\sigma}} \theta_{\sigma}^{G} F_{K, \sigma}^{G}(u), \quad F_{K, \sigma}^{G}(u) \stackrel{\text { def }}{=} \mathrm{m}_{\sigma} \Lambda_{K}\left(\nabla_{\mathcal{D}} u\right)_{K}^{G, \sigma} \cdot \mathbf{n}_{K, \sigma}
$$

Since the subfluxes $F_{K, \sigma}^{G}$ are conservative (second equation in (3.1)), the whole fluxes $F_{K, \sigma}$ are also conservative. Specific methods are obtained from (3.6) by defining a suitable criterion to compute the family of weights $\left\{\theta_{\sigma}^{G}\right\}_{\sigma \in \mathcal{E}, G \in \mathcal{G}_{\sigma}}$.

\subsection{The $\mathbf{L}$ method}

The MPFA L method can be obtained as follows. For all $\sigma \in \mathcal{E}$ and for each $s \in \mathcal{V} \cap \sigma$ we choose one group $G_{\sigma, s} \subset \mathcal{E}_{s}$ according to the criterion of [3] for which $\theta_{\sigma}^{G_{\sigma, s}}=1 / \operatorname{card}(\{\mathrm{s} \in \mathcal{V} \mid \mathrm{s} \in \sigma\})$. The other groups containing $s$ do not contribute to the flux through $\sigma$. This choice was originally intended to improve the monotonicity of the method on parallelogram meshes.

\subsection{The G method}

The alternative choice used in the numerical examples of Section 4 is designed so as to enhance the coercivity of the method. For each group $G \in \mathcal{G}$, define the vector space $\mathcal{H}_{\mathcal{T}_{G}} \stackrel{\text { def }}{=} \mathbb{R}^{\text {card }\left(\mathcal{T}_{G}\right)}$ and denote the components of a generic $v \in \mathcal{H}_{\mathcal{T}_{G}}$ by $\left\{v_{K}\right\}_{K \in \mathcal{T}_{G}}$. The space $\mathcal{H}_{\mathcal{T}_{G}}$ is endowed with the semi-norm

$$
\|u\|_{\mathcal{T}_{G}}^{2} \stackrel{\text { def }}{=} \sum_{K \in \mathcal{T}_{G}} \sum_{\sigma \in \mathcal{E}_{K} \cap G} \frac{\mathrm{m}_{\sigma}}{d_{K, \sigma}}\left(\gamma_{\sigma} u-u_{K}\right)^{2} .
$$

For all $(u, v) \in\left[\mathcal{H}_{\mathcal{T}_{G}}\right]^{2}$ set $a_{\mathcal{T}_{G}}(u, v)=\sum_{K \in \mathcal{T}_{G}} \sum_{\sigma \in \mathcal{E}_{K} \cap G} F_{K, \sigma}^{G}(u)\left(\gamma_{\sigma} v-v_{K}\right)$. For each $G \in \mathcal{G}$ define

$$
\gamma_{2} \stackrel{\text { def }}{=} \inf _{\left\{u \in \mathcal{H}_{\mathcal{T}_{G}},\|u\|_{\mathcal{T}_{G}}=1\right\}} a_{\mathcal{T}_{G}}(u, u) .
$$

The computation of the parameter $\gamma_{2}$ requires to evaluate the eigenvalues of a local matrix of $\mathbb{R}^{d, d}$ associated with the bilinear form $a_{\mathcal{T}_{G}}$, and its cost is negligible. Indeed, by conservativity of the subfluxes,

$$
\begin{aligned}
a_{\mathcal{T}_{G}}(u, u) & =\sum_{\sigma \in G} \sum_{K \in \mathcal{T}_{\sigma}} F_{K, \sigma}^{G}(u)\left(\gamma_{\sigma} u-u_{K}\right) \\
& =\sum_{\sigma \in G \cap \mathcal{E}_{\mathrm{int}}, \mathcal{T}_{\sigma}=\left\{K_{G}, L\right\}} F_{K_{G}, \sigma}^{G}(u)\left(u_{L}-u_{K_{G}}\right)+\sum_{\sigma \in G \cap \mathcal{E}_{\mathrm{ext}}, \mathcal{T}_{\sigma}=\left\{K_{G}\right\}} F_{K_{G}, \sigma}^{G}(u)\left(\gamma_{\sigma} u-u_{K_{G}}\right) .
\end{aligned}
$$


For all $\sigma \in \mathcal{E}_{\text {int }}$ with $\mathcal{T}_{\sigma}=\left\{K_{G}, L\right\}$, it holds $u_{L}-u_{K_{G}}=\frac{d_{K_{G}, \sigma}+d_{L, \sigma}}{d_{K_{G}, \sigma}}\left(\gamma_{\sigma} u-u_{K_{G}}\right)$. Let

$$
d_{\sigma} \stackrel{\text { def }}{=} \begin{cases}d_{K_{G}, \sigma}+d_{L, \sigma} & \forall \sigma \in \mathcal{E}_{\text {int }} \text { with } \mathcal{T}_{\sigma}=\left\{K_{G}, L\right\} \\ d_{K_{G}, \sigma} & \forall \sigma \in \mathcal{E}_{\text {ext }} .\end{cases}
$$

We infer that

$$
a_{\mathcal{T}_{G}}(u, u)=\sum_{\sigma \in G} \frac{d_{\sigma}}{d_{K_{G}, \sigma}} F_{K_{G}, \sigma}^{G}(u)\left(\gamma_{\sigma} u-u_{K_{G}}\right) .
$$

Now, by (3.3), $F_{K_{G}, \sigma}^{G}(u)$ depends linearly on $\left\{u_{L}-u_{K_{G}}\right\}_{L \in \mathcal{T}_{G} \backslash\left\{K_{G}\right\}}$ (and on $u_{K_{G}}$ if $\sigma \in \mathcal{E}_{\text {ext }}$ ). Therefore, we can write

$$
F_{K_{G}, \sigma}^{G}(u)=\sum_{\sigma^{\prime} \in G} a_{\sigma, \sigma^{\prime}}^{G} \frac{d_{\sigma^{\prime}}}{d_{K_{G}, \sigma^{\prime}}}\left(\gamma_{\sigma^{\prime}} u-u_{K_{G}}\right),
$$

for a suitable family of reals $\left\{a_{\sigma, \sigma^{\prime}}^{G}\right\}_{\sigma, \sigma^{\prime} \in G \times G}$. As a consequence,

$$
a_{\mathcal{T}_{G}}(u, u)=\sum_{\left(\sigma, \sigma^{\prime}\right) \in G \times G} \frac{d_{\sigma}}{d_{K_{G}, \sigma}} \frac{d_{\sigma^{\prime}}}{d_{K_{G}, \sigma^{\prime}}} a_{\sigma, \sigma^{\prime}}^{G}\left(\gamma_{\sigma^{\prime}} u-u_{K_{G}}\right)\left(\gamma_{\sigma} u-u_{K_{G}}\right) .
$$

Denote by $X^{G}(u)$ the vector of size $d$ defined by the family $\left\{\frac{\sqrt{d_{\sigma} \mathrm{m}_{\sigma}}}{d_{K_{G}, \sigma}}\left(\gamma_{\sigma} u-u_{K_{G}}\right)\right\}_{\sigma \in G}$ and by $A^{G}$ the matrix of size $d$ defined by the family of reals $\left\{\sqrt{\frac{d_{\sigma} d_{\sigma^{\prime}}}{\mathrm{m}_{\sigma} \mathrm{m}_{\sigma^{\prime}}}} a_{\sigma, \sigma^{\prime}}^{G}\right\}_{\sigma, \sigma^{\prime} \in G \times G}$. Expression (3.7) can be rewritten as

$$
a_{\mathcal{T}_{G}}(u, u)=\left(A^{G} X^{G}(u)\right) \cdot X^{G}(u)=\left(\frac{A^{G}+\left(A^{G}\right)^{t}}{2} X^{G}(u)\right) \cdot X^{G}(u)
$$

where $\left(A^{G}\right)^{t}$ denotes transpose of $A^{G}$. From (3.8), we deduce that $\gamma_{2}$ is the smallest eigenvalue of the matrix $\frac{A^{G}+\left(A^{G}\right)^{t}}{2}$ (indeed, the Euclidean norm of the vector $X^{G}(u)$ is exactly equal to $\|u\|_{\mathcal{T}_{G}}$ ). For a given $\epsilon>0$, let

$$
\begin{cases}g_{\epsilon}(x)=\frac{\epsilon^{2}}{\epsilon-x} & \text { if } x<0, \\ g_{\epsilon}(x)=x+\epsilon & \text { otherwise }\end{cases}
$$

and, for all $G \in \mathcal{G}$, define $\beta^{G}=g_{\epsilon}\left(\gamma_{2}\right)$. The weights are defined as

$$
\theta_{\sigma}^{G}=\frac{\beta^{G}}{\sum_{G^{\prime} \in \mathcal{G}_{\sigma}} \beta^{G^{\prime}}} \quad \forall G \in \mathcal{G}, \forall \sigma \in G
$$

Therefore, for a given $G \in \mathcal{G}$, the larger $\gamma_{2}$, the more the subfluxes $\left\{F_{K, \sigma}^{G}\right\}_{K \in \mathcal{T}_{G}, \sigma \in \mathcal{E}_{K} \cap G}$ will contribute to the global fluxes $\left\{F_{K, \sigma}\right\}_{K \in \mathcal{T}}, \sigma \in \mathcal{E}_{K} \cap G$.

\subsection{Convergence}

Property (P2) is a crucial ingredient for the analysis of Section 2.2, since it allows to prove the existence and uniqueness of a discrete solution as well as to obtain a stability estimate on the discrete solution for use in Theorem 2.2. Unfortunately, proving (P2) for completely general meshes and diffusion tensors is usually not possible for nonsymmetric schemes like the G method. In this section we propose a computable sufficient criterion to check that (P2) holds. This criterion is not optimal in the sense that coercivity may hold even if the assumptions of Lemma 3.4 are violated. For a given discretization and diffusion tensor, this assumption can be 
checked numerically by computing the eigenvalues of a small linear system of $\operatorname{size} \operatorname{card}\left(\mathcal{E}_{s}\right) \leq \varrho_{3}$ for each vertex $s \in \mathcal{V}$. Let $s \in \mathcal{V}$, and set $\mathcal{H}_{\mathcal{T}_{s}} \stackrel{\text { def }}{=}\left\{u_{K} \in \mathbb{R}, K \in \mathcal{T}_{s}\right\}$. The space $\mathcal{H}_{\mathcal{T}_{s}}$ is endowed with the semi-norm

$$
\|u\|_{\mathcal{T}_{s}}^{2} \stackrel{\text { def }}{=} \sum_{K \in \mathcal{T}_{s}} \sum_{\sigma \in \mathcal{E}_{s} \cap \mathcal{E}_{K}} \frac{\mathrm{m}_{\sigma}}{d_{K, \sigma}}\left(\gamma_{\sigma} u-u_{K}\right)^{2} .
$$

Denote by $a_{\mathcal{T}_{s}}$ the bilinear form defined as follows: For all $u, v \in \mathcal{H}_{\mathcal{T}_{s}}$,

$$
a_{\mathcal{T}_{s}}(u, v) \stackrel{\text { def }}{=} \sum_{G \in \mathcal{G}, G \subset \mathcal{E}_{s}} \sum_{K \in \mathcal{T}_{G}} \sum_{\sigma \in \mathcal{E}_{K} \cap G} \theta_{\sigma}^{G} F_{K, \sigma}^{G}(u)\left(\gamma_{\sigma} v-v_{K}\right) .
$$

Lemma 3.4. Let there be a positive constant $\gamma_{3}$ s.t.

$$
\min _{s \in \mathcal{V}} \inf _{\left\{v \in \mathcal{H}_{\mathcal{T}_{s}} \mid\|v\|_{\mathcal{T}_{s}}=1\right\}} a_{\mathcal{T}_{s}}(v, v) \geq \gamma_{3}
$$

Then, for all $u \in \mathcal{H}_{\mathcal{T}}, a_{\mathcal{T}}(u, u) \geq \gamma_{3}\|u\|_{\mathcal{T}}^{2}$.

Proof. For all $u \in \mathcal{H}_{\mathcal{T}}$ and $s \in \mathcal{V}$, let $u_{s} \stackrel{\text { def }}{=}\left(u_{K}\right)_{K \in \mathcal{T}_{s}} \in \mathcal{H}_{\mathcal{T}_{s}}$. Since any given group $G$ only belongs to one particular $\mathcal{E}_{s}$, it is easy to see that $a_{\mathcal{T}}(u, u)=\sum_{s \in \mathcal{V}} a_{\mathcal{T}_{s}}\left(u_{s}, u_{s}\right)$, and thus that $a_{\mathcal{T}}(u, u) \geq \gamma_{3} \sum_{s \in \mathcal{V}}\left\|u_{s}\right\|_{\mathcal{T}_{s}}^{2}$. The assertion then follows from

$$
\sum_{s \in \mathcal{V}}\left\|u_{s}\right\|_{\mathcal{T}_{s}}^{2} \geq\|u\|_{\mathcal{T}}^{2}
$$

which is straightforward since, for all $K \in \mathcal{T}$ and for all $\sigma \in \mathcal{E}_{K}, \operatorname{card}\left(\left\{s \in \mathcal{V} \mid \sigma \in \mathcal{E}_{s}\right\}\right) \geq 1$.

Theorem 3.1 (convergence). Let $\left\{\mathcal{D}_{n}\right\}_{n \in \mathbb{N}}$ be a family of meshes matching Definitions 2.1 and 3.1 and s.t. $h_{\mathcal{D}_{n}} \rightarrow 0$ as $n \rightarrow \infty$. Suppose, furthermore, that (3.9) holds with $\gamma_{3}$ not depending on $n \in \mathbb{N}$ and that there exists $\gamma_{4}<+\infty$ s.t.

$$
\forall n \in \mathbb{N}, \forall \sigma \in \mathcal{E}_{n}, \sum_{G \in \mathcal{G}_{\sigma}} \theta_{\sigma}^{G}\left|\mathcal{A}_{G}^{-1}\right| \leq \gamma_{4} .
$$

Then, as $n \rightarrow \infty$, the sequence $\left\{u_{n}\right\}_{n \in \mathbb{N}}$ of discrete solutions of problem (2.5) with numerical fluxes defined by (3.6) converges to the solution $\bar{u}$ of $(2.1)$ in $L^{q}(\Omega)$ for all $q \in\left[1,2 d /(d-2)\right.$ ) (and weakly in $L^{2 d /(d-2)}(\Omega)$ if $d>2)$.

Proof. It suffices to verify the requirements listed in Assumption 2.1. According to Lemma 3.2 , the choice $\mathfrak{D}=\mathcal{Q}$ meets (P1). The property (P2) holds (with $I_{\sigma}=\gamma_{\sigma}$ ) under (3.9), by Lemma 3.4. Finally, the consistency of the fluxes (P3) can be obtained by proving the strong consistency (see Rem. 2.3). Indeed, for all $n \in \mathbb{N}, K \in \mathcal{T}_{n}$ and $\sigma \in \mathcal{E}_{K},(3.6),(3.5)$, Lemma 3.3 and (3.10) yield

$$
\begin{aligned}
\left|F_{K, \sigma}\left(\varphi_{\mathcal{T}_{n}}\right)-\frac{1}{\mathrm{~m}_{K}} \int_{K} \Lambda(x) \nabla \varphi(x) \cdot \mathrm{m}_{\sigma} \mathbf{n}_{K, \sigma}\right| \leq & \left|F_{K, \sigma}\left(\varphi_{\mathcal{T}_{n}}\right)-\Lambda_{K} \nabla \varphi\left(x_{K}\right) \mathrm{m}_{\sigma} \mathbf{n}_{K, \sigma}\right| \\
& +\left|\frac{1}{\mathrm{~m}_{K}} \int_{K} \Lambda(x)\left(\nabla \varphi(x)-\nabla \varphi\left(x_{K}\right)\right) \cdot \mathrm{m}_{\sigma} \mathbf{n}_{K, \sigma}\right| \\
\leq & \mathrm{m}_{\sigma} \beta_{0} \sum_{G \in \mathcal{G}_{\sigma}} \theta_{\sigma}^{G}\left|\left(\nabla_{\mathcal{D}_{n}} \varphi_{\mathcal{T}_{n}}\right)_{K}^{G, \sigma}-\nabla \varphi\left(x_{K}\right)\right| \\
& +\mathrm{m}_{\sigma} \beta_{0} \sup _{x \in K}\left|\nabla \varphi(x)-\nabla \varphi\left(x_{K}\right)\right| \\
\leq & \left(C_{5} \gamma_{4}+C_{3}\right) \mathrm{m}_{\sigma} \beta_{0} h_{\mathcal{D}_{n}},
\end{aligned}
$$

where $C_{3}=\sup _{x \in P_{\Omega}}\left|\varphi^{\prime \prime}(x)\right|$. 


\subsection{Convergence of the gradient reconstruction}

In order to prove the convergence of the gradient reconstruction (2.15), Assumption 2.2 must be verified; this can be achieved by adding a rather benign assumption on the mesh families.

Assumption 3.2. $\left\{\mathcal{D}_{n}\right\}_{n \in \mathbb{N}}$ is a mesh family matching Definition 2.1 and there exists a non-negative constant $\varrho_{4}$ independent of $n$ s.t.

$$
\max _{K \in \mathcal{I}_{n}} \max _{\sigma \in \mathcal{E}_{K}} \frac{\operatorname{diam}(K)^{d-1}}{\mathrm{~m}_{\sigma}} \leq \varrho_{4} .
$$

This assumption and (2.2) allow to uniformly bound the cardinals of $\mathcal{G}_{\sigma}$. Indeed, since each cell $K$ is starshaped with respect to $x_{K}$ we have, for all $\sigma \in \mathcal{E}_{K}$ and all $x \in \sigma,\left(x-x_{K}\right) \cdot \mathbf{n}_{K, \sigma}=d_{K, \sigma} \geq \varrho_{1} \operatorname{diam}(K)$ and thus, by Stokes' formula,

$$
\begin{aligned}
d \mathrm{~m}_{K}=\int_{K} \operatorname{div}\left(x-x_{K}\right) \mathrm{d} x & =\sum_{\sigma \in \mathcal{E}_{K}} \int_{\sigma}\left(x-x_{K}\right) \cdot \mathbf{n}_{K, \sigma} \\
& \geq \varrho_{1} \operatorname{diam}(K) \sum_{\sigma \in \mathcal{E}_{K}} \mathrm{~m}_{\sigma} \geq \frac{\varrho_{1}}{\varrho_{4}} \operatorname{diam}(K)^{d} \operatorname{card}\left(\mathcal{E}_{K}\right) .
\end{aligned}
$$

As $\mathrm{m}_{K} \leq \omega_{d} \operatorname{diam}(K)^{d}, \omega_{d}$ being the volume of the unit ball in $\mathbb{R}^{d}$, this shows that $\operatorname{card}\left(\mathcal{E}_{K}\right) \leq \frac{d \omega_{d} \varrho_{4}}{\varrho_{1}}$; but, if $\mathcal{T}_{\sigma}=\{K, L\}, \mathcal{G}_{\sigma}$ is contained in the set of families of $d$ faces chosen in $\mathcal{E}_{K}$ or $\mathcal{E}_{L}$, and there thus exists $C_{6}$ only depending on $\varrho_{4}, \varrho_{1}$ s.t.

$$
\max _{\sigma \in \mathcal{E}_{n}} \operatorname{card}\left(\mathcal{G}_{\sigma}\right) \leq C_{6} .
$$

Lemma 3.5. Let $\left\{\mathcal{D}_{n}\right\}_{n \in \mathbb{N}}$ be a family of discretizations matching Definition 2.1 and 3.1 and assume that Assumption 3.2 and (3.10) hold. Then, Assumption 2.2 holds.

Proof. For simplicity of notation, the subscript $n$ will be suppressed throughout the proof, which holds for a generic element of the mesh family $\left\{\mathcal{D}_{n}\right\}_{n \in \mathbb{N}}$. Let $v \in H_{\mathcal{T}}(\Omega)$.

(i) For $G \in \mathcal{G}$, we estimate $\left|\left(\nabla_{\mathcal{D}} v\right)_{K_{G}}^{G}\right|$. Since $\left(\nabla_{\mathcal{D}} v\right)_{K_{G}}^{G}$ solves (3.3), and recalling that $\gamma_{\sigma} v=0$ for all $\sigma \in \mathcal{E}_{\text {ext }}$,

$$
\left|\left(\nabla_{\mathcal{D}} v\right)_{K_{G}}^{G}\right|^{2} \leq\left|\mathcal{A}_{G}^{-1}\right|^{2} \beta_{0}^{2}\left[\sum_{\sigma \in G \cap \mathcal{E}_{\mathrm{int}}, \mathcal{T}_{\sigma}=\left\{K_{G}, L\right\}} \frac{\left(v_{L}-v_{K_{G}}\right)^{2}}{d_{L, \sigma}^{2}}+\sum_{\sigma \in G \cap \mathcal{E}_{\mathrm{ext}}} \frac{\left(\gamma_{\sigma} v-v_{K_{G}}\right)^{2}}{d_{K_{G}, \sigma}^{2}}\right] .
$$

Observe that, for all $\sigma \in \mathcal{E}_{\text {int }}$, denoting $\mathcal{T}_{\sigma}=\{K, L\}$ we have $\frac{v_{L}-v_{K}}{d_{K, \sigma}+d_{L, \sigma}}=\frac{\gamma_{\sigma} v-v_{K}}{d_{K, \sigma}}$. As a consequence, by (2.2),

$$
\frac{\left(v_{L}-v_{K}\right)^{2}}{d_{L, \sigma}^{2}} \leq \frac{\left(\gamma_{\sigma} v-v_{K}\right)^{2}}{d_{K, \sigma}^{2}}\left(1+\frac{1}{\varrho_{2}}\right)^{2}
$$

and

$$
\mathrm{m}_{K_{G}}\left|\left(\nabla_{\mathcal{D}} v\right)_{K_{G}}^{G}\right|^{2} \leq\left|\mathcal{A}_{G}^{-1}\right|^{2} \beta_{0}^{2}\left(1+\frac{1}{\varrho_{2}}\right)^{2} \sum_{\sigma \in G} \frac{\mathrm{m}_{K_{G}}}{d_{K_{G}, \sigma}^{2}}\left(\gamma_{\sigma} v-v_{K_{G}}\right)^{2} .
$$

(ii) For $G \in \mathcal{G}, \sigma \in G \cap \mathcal{E}_{\text {int }}$ with $\mathcal{T}_{\sigma}=\left\{K_{G}, L\right\}$, we estimate $\left|\left(\nabla_{\mathcal{D}} v\right)_{L}^{G, \sigma}\right|$. Owing to (3.4),

$$
\left|\left(\nabla_{\mathcal{D}} v\right)_{L}^{G, \sigma}\right|^{2} \leq 2\left[\left|\left(\nabla_{\mathcal{D}} v\right)_{K_{G}}^{G}\right|^{2}\left(1+\frac{\left|x_{L}-x_{K_{G}}\right|}{d_{L, \sigma}}\right)^{2}+\frac{\left|v_{L}-v_{K_{G}}\right|^{2}}{d_{L, \sigma}^{2}}\right] .
$$


Using (2.2) and observing that $\left|x_{L}-x_{K_{G}}\right| \leq \operatorname{diam}(L)+\operatorname{diam}\left(K_{G}\right)$, we have that

$$
\frac{\left|x_{L}-x_{K_{G}}\right|}{d_{L, \sigma}} \leq \frac{1}{\varrho_{1}}\left(1+\frac{1}{\varrho_{2}}\right)
$$

Then, from (3.14) together with (3.13), (3.15) and (3.12), we deduce that there exists $C_{7}>0$ which solely depends on $\beta_{0}, \varrho_{1}$ and $\varrho_{2}$ s.t.

$$
\mathrm{m}_{L}\left|\left(\nabla_{\mathcal{D}} v\right)_{L}^{G, \sigma}\right|^{2} \leq C_{7}\left(1+\left|\mathcal{A}_{G}^{-1}\right|^{2}\right) \frac{\mathrm{m}_{L}}{\mathrm{~m}_{K_{G}}} \sum_{\sigma^{\prime} \in G} \frac{\mathrm{m}_{K_{G}}}{d_{K_{G}, \sigma^{\prime}}^{2}}\left(\gamma_{\sigma^{\prime}} v-v_{K_{G}}\right)^{2} .
$$

(iii) Stability. The fact that $K$ is star-shaped with respect to $x_{K}$ and the definition of $d_{K, \sigma}$ show that $K$ contains the ball centered at $x_{K}$ and with radius $\inf _{\sigma \in \mathcal{E}_{K}} d_{K, \sigma}$; we deduce from (2.2) that $C_{8} \operatorname{diam}(K)^{d} \leq \mathrm{m}_{K} \leq$ $C_{9} \operatorname{diam}(K)^{d}$ (with $C_{8}$ and $C_{9}$ only depending on $\varrho_{1}$ ) and thus that, if $K$ and $L$ are two neighboring grid cells, there exists $C_{10}>0$ only depending on $\varrho_{2}$ and $\varrho_{1}$ s.t. $\frac{\mathrm{m}_{L}}{\mathrm{~m}_{K}} \leq C_{10}$. Hence, thanks to (3.13) and (3.16), there exists a real $C_{11}>0$ solely depending on $\varrho_{1}, \varrho_{2}$ and $\beta_{0}$ s.t.

$$
\forall \sigma \in G, \forall K \in \mathcal{T}_{\sigma}, \quad \mathrm{m}_{K}\left|\left(\nabla_{\mathcal{D}} v\right)_{K}^{G, \sigma}\right|^{2} \leq C_{11}\left(1+\left|\mathcal{A}_{G}^{-1}\right|^{2}\right) \sum_{\sigma^{\prime} \in G} \frac{\mathrm{m}_{K_{G}}}{d_{K_{G}, \sigma^{\prime}}^{2}}\left(\gamma_{\sigma^{\prime}} v-v_{K_{G}}\right)^{2} .
$$

Furthermore, observe that, for all $\sigma^{\prime} \in G, K_{G}$ belongs to $\mathcal{T}_{\sigma^{\prime}}$, so that

$$
\frac{\mathrm{m}_{K_{G}}}{d_{K_{G}, \sigma^{\prime}}^{2}}\left(\gamma_{\sigma^{\prime}} v-v_{K_{G}}\right)^{2} \leq \sum_{K \in \mathcal{T}_{\sigma^{\prime}}} \frac{\mathrm{m}_{K}}{d_{K, \sigma^{\prime}}^{2}}\left(\gamma_{\sigma^{\prime}} v-v_{K}\right)^{2} .
$$

We infer from (2.3) and the Definition (3.6) of the fluxes that

$$
\begin{aligned}
T \stackrel{\text { def }}{=} \sum_{K \in \mathcal{T}} \sum_{\sigma \in \mathcal{E}_{K}} \frac{d_{K, \sigma}}{\mathrm{m}_{\sigma}}\left|F_{K, \sigma}(v)\right|^{2} & =\sum_{K \in \mathcal{T}} \sum_{\sigma \in \mathcal{E}_{K}} \frac{d_{K, \sigma}}{\mathrm{m}_{\sigma}}\left|\mathrm{m}_{\sigma} \Lambda_{K} \sum_{G \in \mathcal{G}_{\sigma}} \theta_{\sigma}^{G}\left(\nabla_{\mathcal{D}} v\right)_{K}^{G, \sigma} \cdot \mathbf{n}_{K, \sigma}\right|^{2} \\
& \leq d \beta_{0}^{2} \sum_{K \in \mathcal{T}} \sum_{\sigma \in \mathcal{E}_{K}} \mathrm{~m}_{K}\left|\sum_{G \in \mathcal{G}_{\sigma}} \theta_{\sigma}^{G}\left(\nabla_{\mathcal{D}} v\right)_{K}^{G, \sigma} \cdot \mathbf{n}_{K, \sigma}\right|^{2}
\end{aligned}
$$

Equation (3.10) gives in particular $\theta_{\sigma}^{G}\left|\mathcal{A}_{G}^{-1}\right| \leq \gamma_{4}$ and thus, by Cauchy-Schwarz inequality, (3.11) and (3.17), we deduce

$$
\begin{aligned}
T & \leq d \beta_{0}^{2} \sum_{K \in \mathcal{T}} \sum_{\sigma \in \mathcal{E}_{K}} \mathrm{~m}_{K} \sum_{G \in \mathcal{G}_{\sigma}}\left(\theta_{\sigma}^{G}\right)^{2}\left|\left(\nabla_{\mathcal{D}} v\right)_{K}^{G, \sigma}\right|^{2} \times \operatorname{card}\left(\mathcal{G}_{\sigma}\right) \\
& \leq d \beta_{0}^{2} C_{6} C_{11}\left(1+\gamma_{4}^{2}\right) \sum_{K \in \mathcal{T}} \sum_{\sigma \in \mathcal{E}_{K}} \sum_{G \in \mathcal{G}_{\sigma}} \sum_{\sigma^{\prime} \in G} \frac{\mathrm{m}_{K_{G}}}{d_{K_{G}, \sigma^{\prime}}^{2}}\left(\gamma_{\sigma^{\prime}} v-v_{K_{G}}\right)^{2} .
\end{aligned}
$$

We then permute some sums and use the fact that each $\mathcal{T}_{\sigma}$ has one or two elements to obtain $C_{12}$ only depending on $\varrho_{1}, \varrho_{2}, \beta_{0}$ and $\gamma_{4}$ s.t.

$$
\begin{aligned}
T & \leq C_{12} \sum_{\sigma \in \mathcal{E}} \sum_{K \in \mathcal{T}_{\sigma}}\left(\sum_{G \in \mathcal{G}_{\sigma}} \sum_{\sigma^{\prime} \in G} \frac{\mathrm{m}_{K_{G}}}{d_{K_{G}, \sigma^{\prime}}^{2}}\left(\gamma_{\sigma^{\prime}} v-v_{K_{G}}\right)^{2}\right) \\
& \leq 2 C_{12} \sum_{\sigma \in \mathcal{E}} \sum_{G \in \mathcal{G}_{\sigma}} \sum_{\sigma^{\prime} \in G} \frac{\mathrm{m}_{K_{G}}}{d_{K_{G}, \sigma^{\prime}}^{2}}\left(\gamma_{\sigma^{\prime}} v-v_{K_{G}}\right)^{2}=2 C_{12} \sum_{G \in \mathcal{G}} \sum_{\sigma \in G} \sum_{\sigma^{\prime} \in G} \frac{\mathrm{m}_{K_{G}}}{d_{K_{G}, \sigma^{\prime}}^{2}}\left(\gamma_{\sigma^{\prime}} v-v_{K_{G}}\right)^{2} .
\end{aligned}
$$


TABLE 1. Convergence results on the test of Section 4.1.

\begin{tabular}{|c|ccccccc|}
\hline nunkw & nnmat & erfl & order & erl2 & order & umin & umax \\
\hline 56 & 480 & $3.34 \mathrm{e}-01$ & - & $1.87 \mathrm{e}-02$ & - & $4.79 \mathrm{e}-02$ & $9.47 \mathrm{e}-01$ \\
224 & 2080 & $1.67 \mathrm{e}-01$ & $1.00 \mathrm{e}+00$ & $4.85 \mathrm{e}-03$ & $1.95 \mathrm{e}+00$ & $1.27 \mathrm{e}-02$ & $9.89 \mathrm{e}-01$ \\
896 & 8640 & $8.34 \mathrm{e}-02$ & $1.00 \mathrm{e}+00$ & $1.14 \mathrm{e}-03$ & $2.09 \mathrm{e}+00$ & $3.30 \mathrm{e}-03$ & $9.97 \mathrm{e}-01$ \\
3584 & 35200 & $4.17 \mathrm{e}-02$ & $1.00 \mathrm{e}+00$ & $2.75 \mathrm{e}-04$ & $2.05 \mathrm{e}+00$ & $8.33 \mathrm{e}-04$ & $9.99 \mathrm{e}-01$ \\
\hline
\end{tabular}

But each group $G$ has cardinal $d$ and thus, by (3.18),

$$
\begin{aligned}
T & \leq 2 C_{12} d \sum_{G \in \mathcal{G}} \sum_{\sigma^{\prime} \in G} \frac{\mathrm{m}_{K_{G}}}{d_{K_{G}, \sigma^{\prime}}^{2}}\left(\gamma_{\sigma^{\prime}} v-v_{K_{G}}\right)^{2}=2 C_{12} d \sum_{\sigma^{\prime} \in \mathcal{E}} \sum_{G \in \mathcal{G}_{\sigma^{\prime}}} \frac{\mathrm{m}_{K_{G}}}{d_{K_{G}, \sigma^{\prime}}^{2}}\left(\gamma_{\sigma^{\prime}} v-v_{K_{G}}\right)^{2} \\
& \leq 2 C_{12} d \sum_{\sigma^{\prime} \in \mathcal{E}}\left(\sum_{K \in \mathcal{T}_{\sigma^{\prime}}} \frac{\mathrm{m}_{K}}{d_{K, \sigma^{\prime}}^{2}}\left(\gamma_{\sigma^{\prime}} v-v_{K}\right)^{2} \times \operatorname{card}\left(\mathcal{G}_{\sigma^{\prime}}\right)\right) .
\end{aligned}
$$

Assumption 3.2, equation (2.2) and $\mathrm{m}_{K} \leq C_{9} \operatorname{diam}(K)^{d}$ imply that $\frac{\mathrm{m}_{K}}{d_{K, \sigma^{\prime}}^{2}} \leq \frac{C_{9} \varrho_{4}}{\varrho_{1}} \frac{\mathrm{m}_{\sigma^{\prime}}}{d_{K, \sigma^{\prime}}}$ and we thus infer

$$
\sum_{K \in \mathcal{T}} \sum_{\sigma \in \mathcal{E}_{K}} \frac{d_{K, \sigma}}{\mathrm{m}_{\sigma}}\left|F_{K, \sigma}(v)\right|^{2} \leq 2 C_{12} d C_{6} \frac{C_{9} \varrho_{4}}{\varrho_{1}}\|v\|_{\mathcal{T}}^{2}
$$

which concludes the proof.

The following result is a direct consequence of Theorem 2.2 together with Lemma 3.5.

Theorem 3.2. Let $\bar{u}$ be the solution to (2.1). Let $\left\{\mathcal{D}_{n}\right\}_{n \in \mathbb{N}}$ be a family of meshes matching Definitions 2.1 and 3.1 and s.t. $h_{\mathcal{D}_{n}} \rightarrow 0$ as $n \rightarrow \infty$ and denote by $u_{n}$ the solution of (2.5) with numerical fluxes defined by (3.6) on $\mathcal{D}_{n}$. Then, if (P2), (3.10) and Assumption 3.2 hold, the sequence $\left\{\bar{\nabla}_{\mathcal{D}_{n}} u_{n}\right\}_{n \in \mathbb{N}}$ converges to $\nabla \bar{u}$ in $\left[L^{2}(\Omega)\right]^{d}$.

\section{Numerical TESTS}

\subsection{Convergence rates}

In order to numerically evaluate the convergence rate of the method described in Section 3.4, we considered the following exact solution:

$$
\bar{u}=\sin (\pi x) \sin (\pi y), \quad \Lambda=\operatorname{diag}(1,1) .
$$

The corresponding discrete Dirichlet problem with $f=2 \pi^{2} \sin (\pi x) \sin (\pi y)$ was solved on a family of conformal shape regular triangular meshes. The following indicators have been considered: 12err, the $L^{2}$ error; erfl, the error on the fluxes defined as

$$
\operatorname{erfl} \stackrel{\text { def }}{=}\left(\sum_{K \in \mathcal{T}_{n}} \sum_{\sigma \in \mathcal{E}_{K}} \frac{d_{K, \sigma}}{\mathrm{m}_{\sigma}}\left|F_{K, \sigma}(u)-\mathrm{m}_{\sigma} \frac{\int_{K} \Lambda(x) \nabla \bar{u}(x) \mathrm{d} x}{\mathrm{~m}_{K}} \cdot \mathbf{n}_{K, \sigma}\right|^{2}\right)^{\frac{1}{2}} .
$$

Formula (4.1) is inspired by the expression of the consistency error (2.8); nzmat, the number of nonzero matrix entries; umin, the minimum of the discrete solution; umax, the maximum of the discrete solution. The number of degrees of freedom is denoted by nunkw. The results are summarized in Table 1. The G-method yields first order convergence in terms of erfl, while second order convergence was observed for erl2. These results are consistent with the ones obtained with a classical two-point method on $\Lambda$-orthogonal meshes. Here and in the following subsection, the parameter $\epsilon$ introduced in Section 3.4 was take equal to 0.1 . 


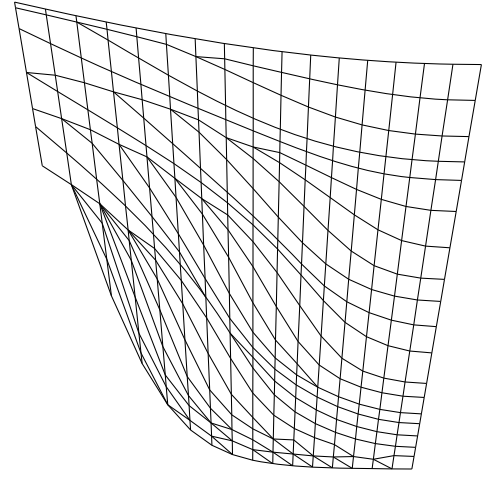

(a) Basin mesh. The actual aspect ratio is 10:1 (x:y).

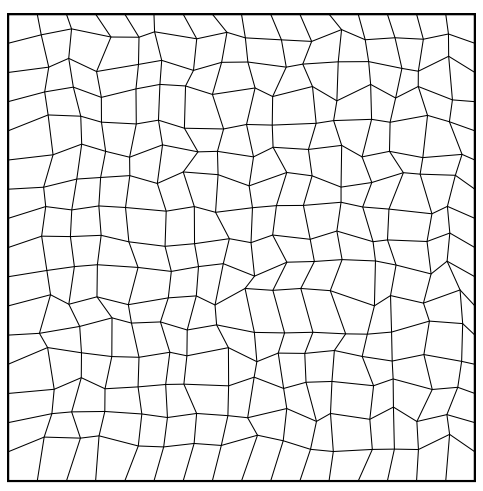

(b) Randomly perturbed quadrangular mesh.

Figure 4. Mesh families.

\subsection{Anisotropic heterogeneous problems}

The objective of this section is to assess the performance of the method described in Section 3.4 on challenging diffusion problems combining mild or strong anisotropy, heterogeneity and distorted or skewed meshes. For the sake of completeness, a comparison is provided against (i) the method of [24], Section 2.2, referred to as Success; (ii) the MPFA O method of [1] and (iii) the MPFA L method of [3,5], also described in Section 3.3. In the first test case (see Figs. 5 and 6 for the numerical results), we consider the Dirichlet problem associated with the following exact solution featuring anisotropic permeability:

$$
\bar{u}=\sin (\pi x) \sin (\pi y), \quad \Lambda=\operatorname{diag}(0.1,1)
$$

In the second test case (see Fig. 7 for the numerical results), we consider the Dirichlet problem associated with the following exact solution featuring heterogeneous anisotropic permeability:

$$
\bar{u}= \begin{cases}\sin (b \pi x) \sin (c \pi y) & \text { if } x \leq \delta \\ \sin (b \pi \delta) \sin (c \pi y)+\pi b \frac{a_{1}}{a_{2}} \cos (b \pi \delta) \sin (c \pi y)(x-\delta) & \text { otherwise }\end{cases}
$$

and

$$
\Lambda=\left\{\begin{array}{lc}
\operatorname{diag}\left(a_{1}, b_{1}\right) & \text { if } x \leq \delta, \\
\operatorname{diag}\left(a_{2}, b_{2}\right) & \text { otherwise }
\end{array}\right.
$$

where $b=\frac{1}{1.7}, c=1.9, a_{1}=1, b_{1}=10, a_{2}=5, b_{2}=1, \delta=0.5$. Both tests have been run on (i) a family of Corner Point Geometry basin meshes with erosion (see Fig. 4(a)) and (ii) a family of randomly distorted quadrangular meshes of $(0,10) \times(0,1)$ (see Fig. $4(\mathrm{~b}))$.

In addition to the indicators of the previous section, the number of preconditioned GMRes iterations nit was also considered. Blown up methods with respect to one indicator are not plotted to keep the scale readable. The linear systems have been solved with a direct solver for the indicators 12err, umin, umax and ergrad, whereas the GMRes algorithm from PETSc [11-13] with Hypre BoomerAMG preconditioner (see www.1lnl.gov/CASC/ hypre) has been used for nit. The stopping criterion required the preconditioned residual norm to be smaller than $10^{-7}$. As expected, while sometimes displaying better accuracy, the Success scheme of [24], Section 2.2, has much denser matrices. 


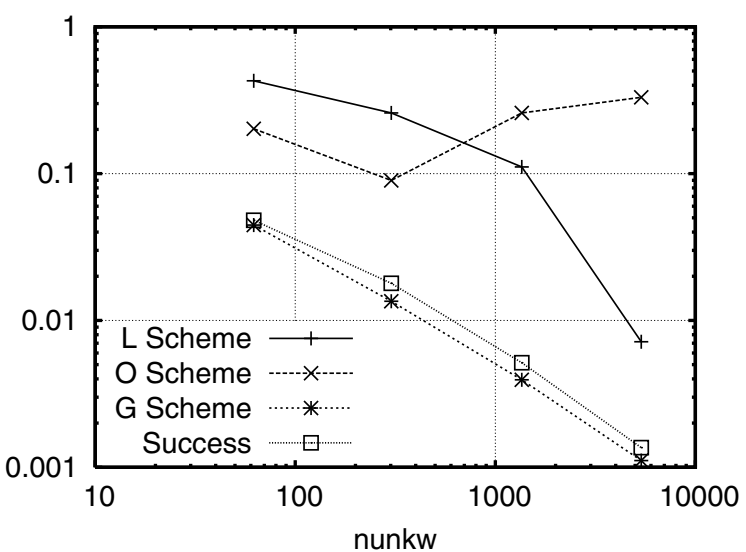

(a) $12 \mathrm{err}$

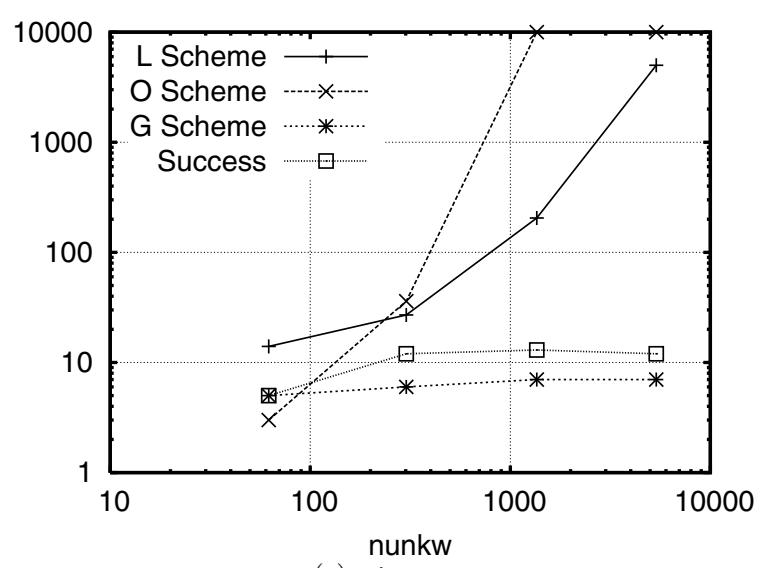

(c) nit

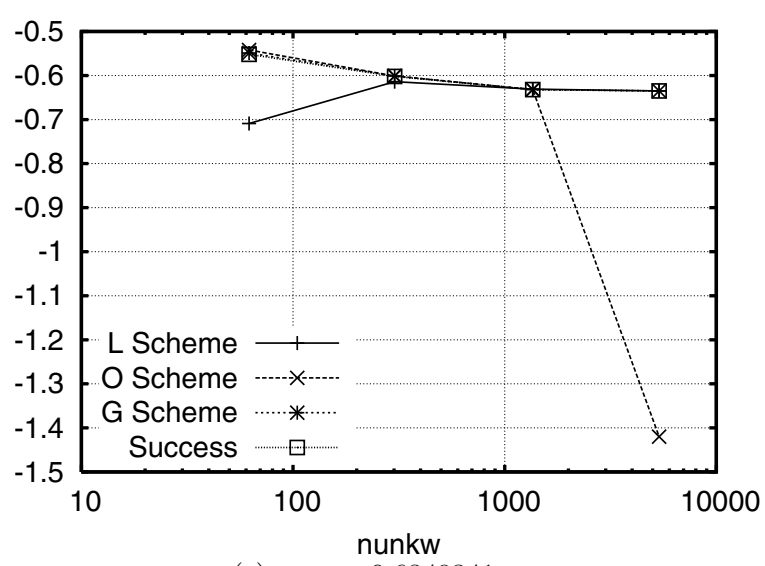

(e) umin $=-0.6349341$

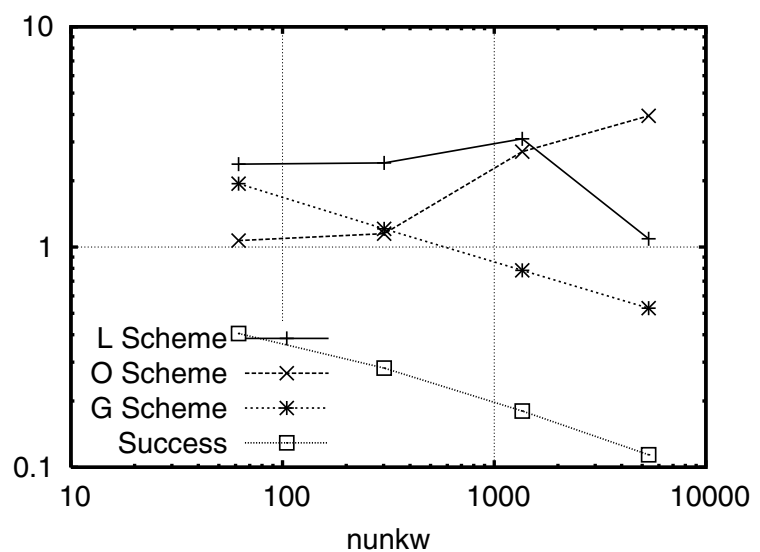

(b) ergrad

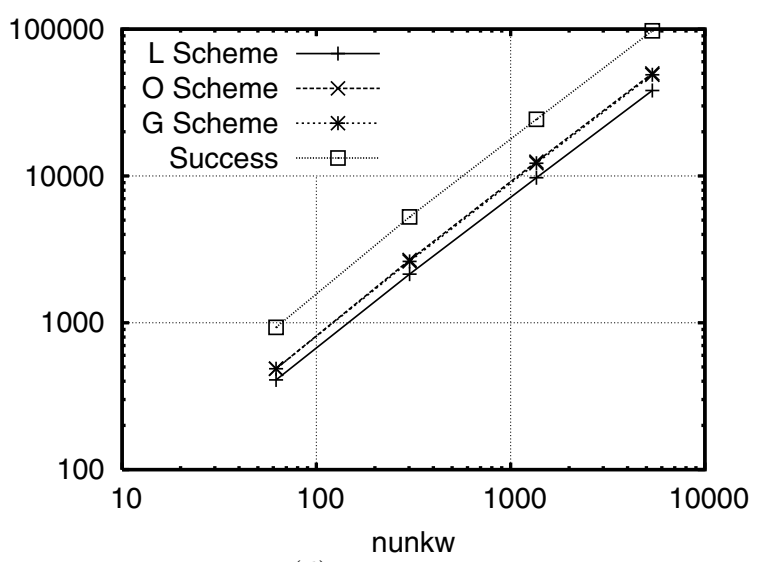

(d) nzmat

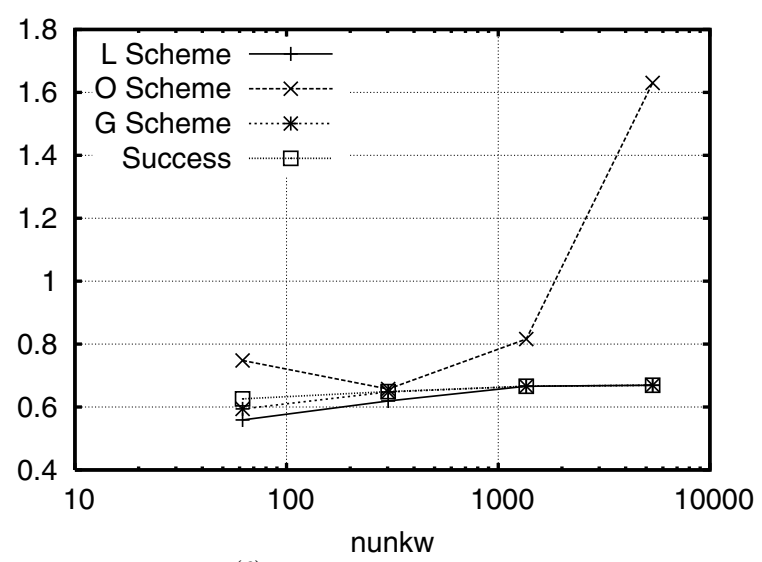

(f) $u \max =0.6687703$

FiguRE 5. Numerical results for test case 1 on the basin mesh family. 


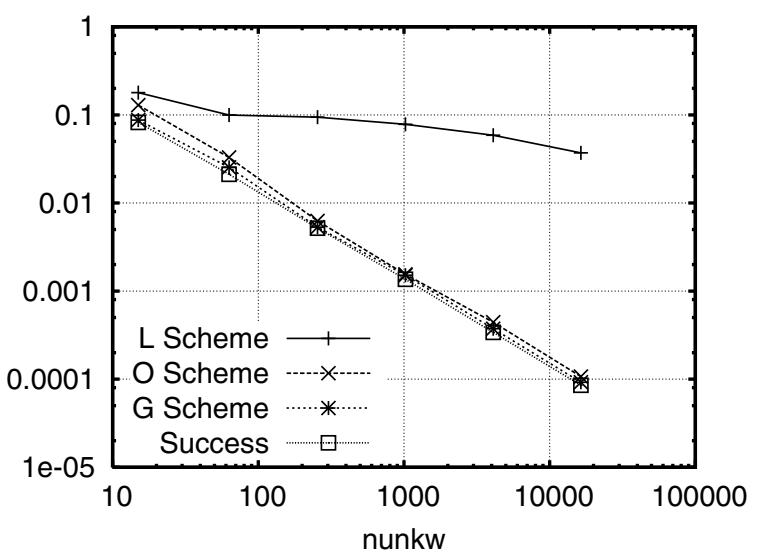

(a) $12 \mathrm{err}$

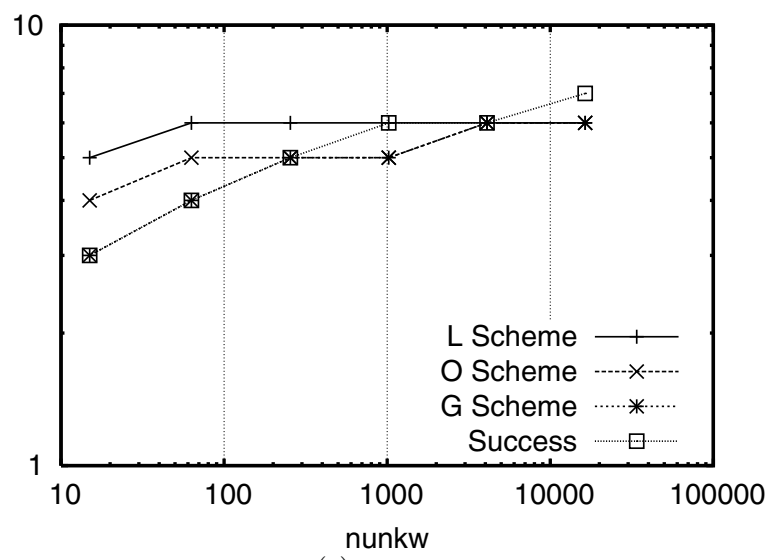

(c) nit

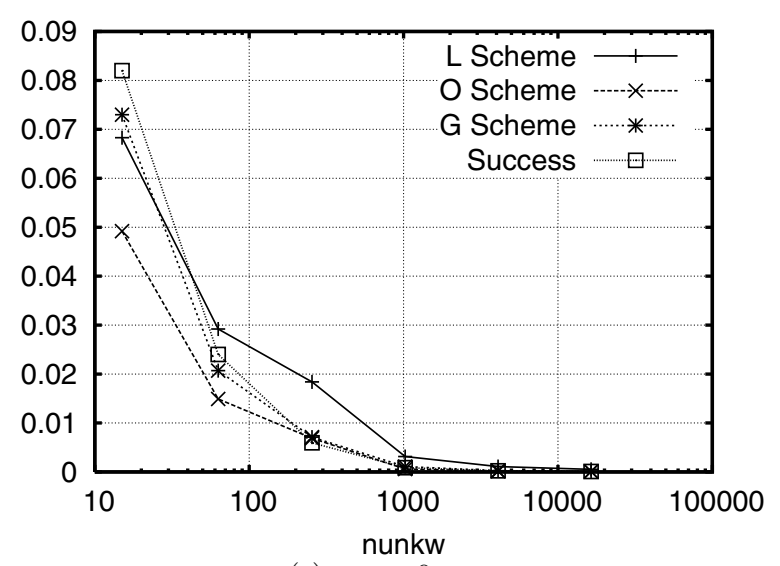

(e) $u \min =0$

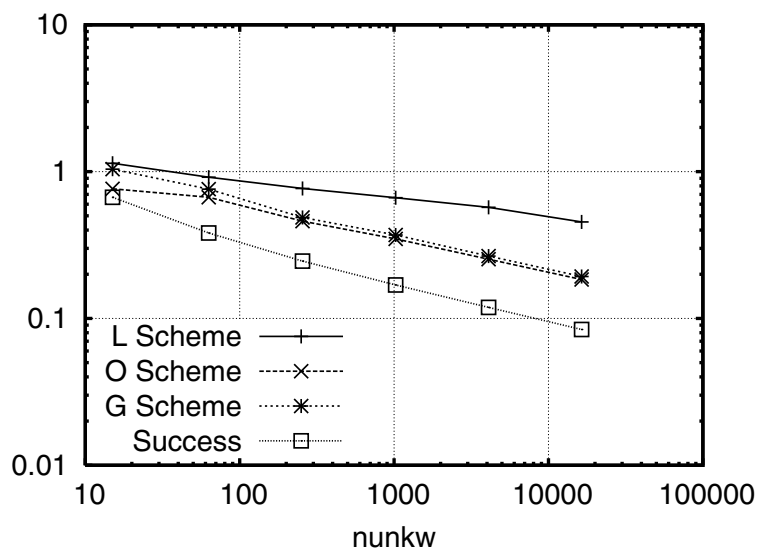

(b) ergrad=1

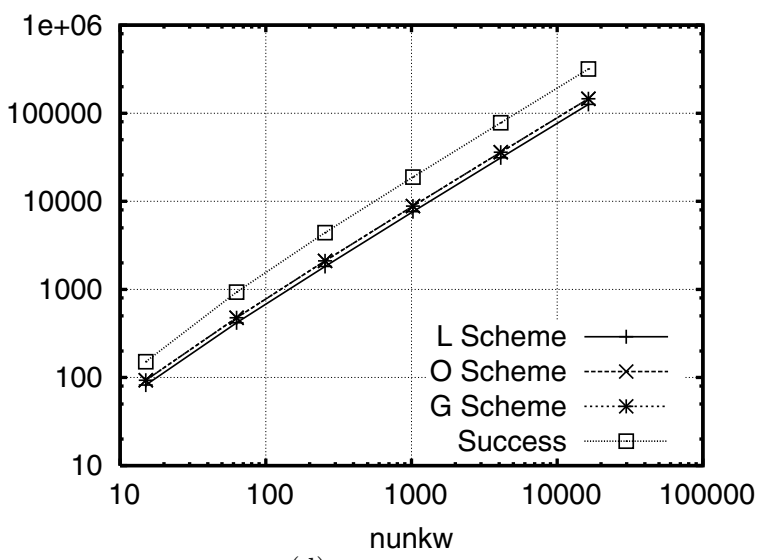

(d) nzmat

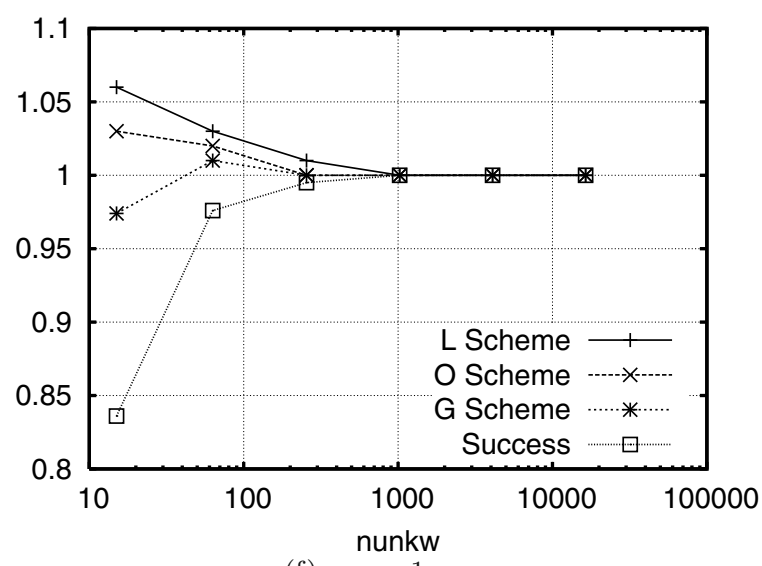

(f) $u \max =1$

FiguRE 6. Numerical results for test case 1 on the randomly perturbed mesh family. 


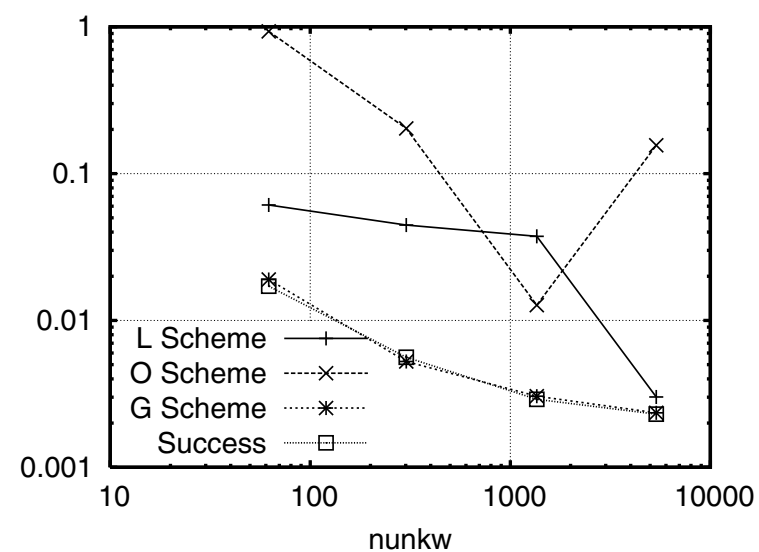

(a) $12 \mathrm{err}$

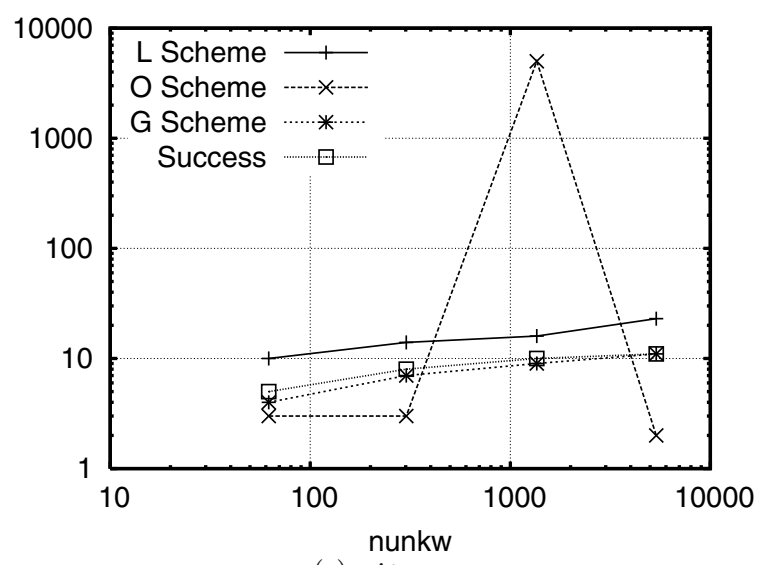

(c) nit

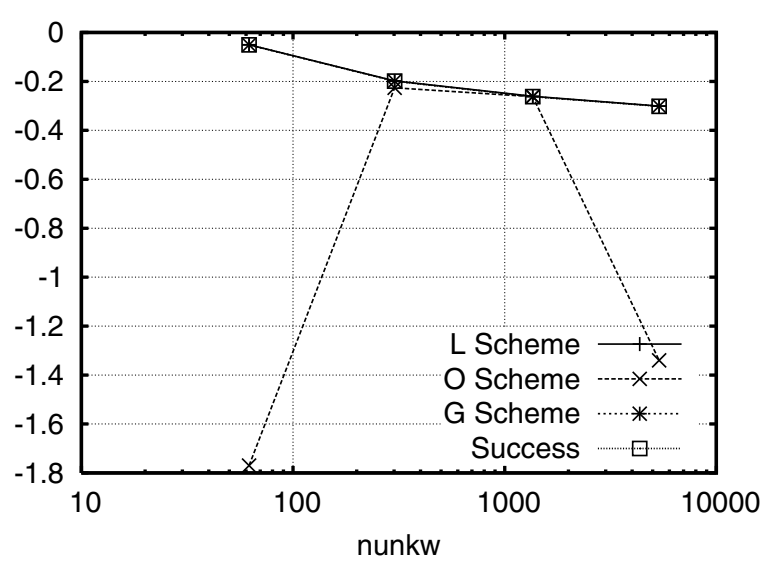

(e) umin $=-0.3013243$

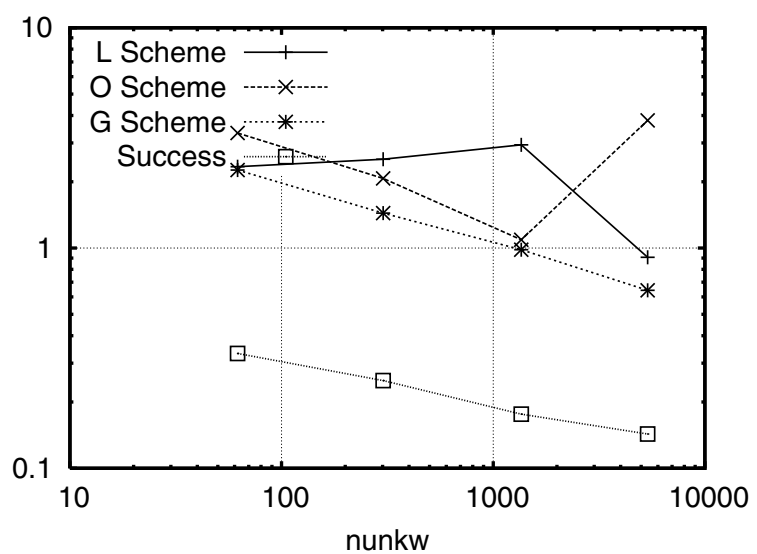

(b) ergrad

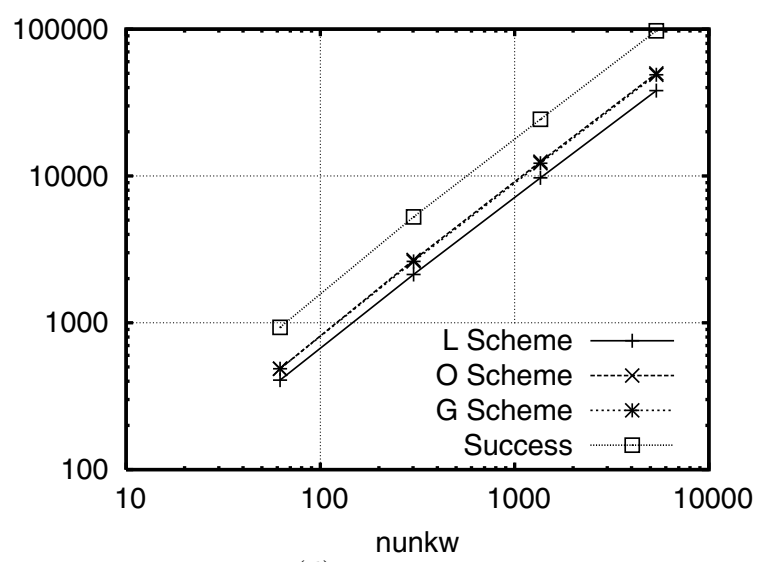

(d) nzmat

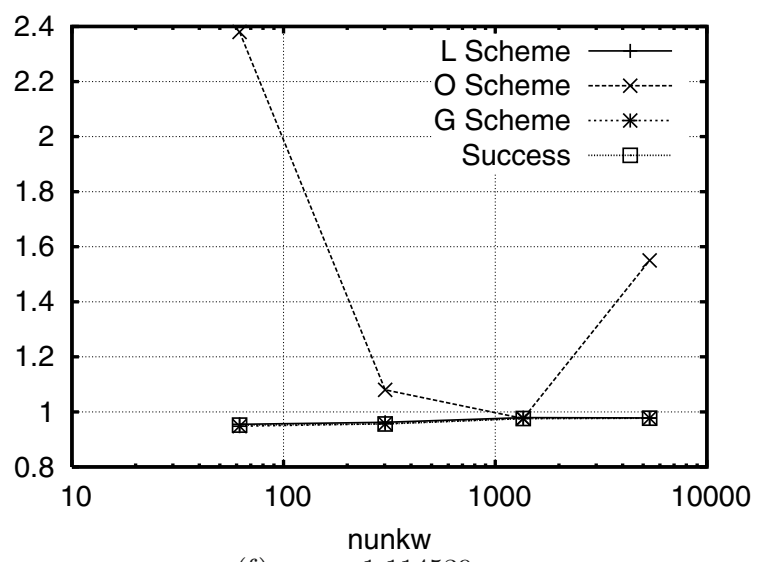

(f) $u \max =1.114539$

FiguRE 7. Numerical results for test case 2 on the basin mesh family. 


\section{A. Proof of Lemma 3.2}

The proof is trivial if $\Lambda \in C(\bar{\Omega})$ since, in this case, $C_{c}^{\infty}(\Omega)$ is contained in $\mathcal{Q}$. The difficulty comes from the possible discontinuities of $\Lambda$ through the interfaces of $P_{\Omega}$, in which case item (iii) of Lemma 3.2 is not easy to obtain and might impose discontinuity of $\nabla \varphi$ through these interfaces. The proof is made in several steps, following the idea of [18]: we first eliminate the singularities (vertices if $d=2$, vertices and edges if $d=3$, etc.) of the boundaries of the open sets $\left\{\Omega_{i}\right\}_{1 \leq i \leq N_{\Omega}}$ by showing that we only need approximate functions which vanish around these singularities; then we reason on each $\overline{\Omega_{i}}$, approximating a given function by functions having the same value on the boundary and vanishing derivatives in the direction $\Lambda \mathbf{n}$; gluing these approximations together, we obtain a function in $\mathcal{Q}$ which is close to the initial given function.

(i) Elimination of the singularities of $\left\{\Omega_{i}\right\}_{1 \leq i \leq N_{\Omega}}$. First of all we notice that, since $C_{c}^{\infty}(\Omega)$ is dense in $H_{0}^{1}(\Omega)$, the result of the lemma follows if we prove that functions in $\mathcal{Q}$ approximate, in $H_{0}^{1}(\Omega)$, any $\psi \in C_{c}^{\infty}(\Omega)$.

Let $S$ be the set of singularities of $\cup_{i=1}^{N_{\Omega}} \partial \Omega_{i}$ (i.e. affine parts of dimension $d-2$ or less: the vertices in dimension $d=2$, the vertices and edges if $d=3$, etc.); it is known that $S$ has a 2-capacity equal to 0 and we can therefore find a sequence of functions $\gamma_{n} \in C_{c}^{\infty}\left(\mathbb{R}^{d} ;[0,1]\right)$ s.t. $\gamma_{n} \rightarrow 0$ in $H^{1}\left(\mathbb{R}^{n}\right)$ as $n \rightarrow \infty$ and, for all $n \in \mathbb{N}, \gamma_{n} \equiv 1$ on a neighborhood of $S$. If $\psi \in C_{c}^{\infty}(\Omega)$ and $\psi_{n}=\left(1-\gamma_{n}\right) \psi \in C_{c}^{\infty}(\Omega)$, then $\psi_{n} \rightarrow \psi$ in $H_{0}^{1}(\Omega)$ and, for all $n, \psi_{n} \equiv 0$ on a neighborhood of $S$. Hence, denoting by $C_{c, S}^{\infty}(\Omega)$ the set of functions in $C_{c}^{\infty}(\Omega)$ which vanish on neighborhoods of $S$, the proof of the lemma is complete if we can approximate, in $H_{0}^{1}(\Omega)$, elements of $C_{c, S}^{\infty}(\Omega)$ by elements of $\mathcal{Q}$.

(ii) Reduction to a $\Omega_{i}$. Let $\psi \in C_{c, S}^{\infty}(\Omega)$ and assume that, for all $1 \leq i \leq N_{\Omega}$, there exists a sequence $\varphi_{n}^{i} \in C^{2}\left(\overline{\Omega_{i}}\right)$ which converges to $\psi$ in $H^{1}\left(\Omega_{i}\right)$ as $n \rightarrow \infty$ and s.t., for all $n \in \mathbb{N}, \varphi_{n}^{i}=\psi$ and $\left(\Lambda \nabla \varphi_{n}^{i}\right)_{\mid \overline{\Omega_{i}}} \cdot \mathbf{n}_{i}=0$ on $\partial \Omega_{i}$. Define then $\varphi_{n}: \bar{\Omega} \rightarrow \mathbb{R}$ as the function equal to $\varphi_{n}^{i}$ on $\overline{\Omega_{i}}$ for all $i=1, \ldots, N_{\Omega} ;$ since $\varphi_{n}^{i}=\varphi_{n}^{j}=\psi$ on $\partial \Omega_{i} \cap \partial \Omega_{j}, \varphi_{n}$ is well defined and continuous on $\bar{\Omega}$, it is $C^{2}$ on each $\overline{\Omega_{i}}$, it vanishes on $\partial \Omega$ (on which $\psi=0$ ) and the tangential derivatives of $\varphi_{n}$ are continuous through the interfaces of $P_{\Omega}$ (for all $\mathbf{t}$ parallel to $\partial \Omega_{i} \cap \partial \Omega_{j}$, the values of $\left(\nabla \varphi_{n}\right)_{\mid \overline{\Omega_{i}}} \cdot \mathbf{t}$ and $\left(\nabla \varphi_{n}\right)_{\mid \overline{\Omega_{j}}} \cdot \mathbf{t}$ on $\partial \Omega_{i} \cap \partial \Omega_{j}$ can be computed using only the values of $\varphi_{n}^{i}=\varphi_{n}^{j}=\psi$ on $\partial \Omega_{i} \cap \partial \Omega_{j}$, and are therefore equal). The continuity of $\varphi_{n}$ across the boundary of $\Omega_{i}$ for each $i$ moreover ensures that $\nabla \varphi_{n}$ has no singularity on these boundaries and it is therefore simply the function equal to $\nabla \varphi_{n}^{i}$ on $\Omega_{i}$ for all $i$; hence, $\varphi_{n} \rightarrow \psi$ in $H_{0}^{1}(\Omega)$. Finally, the fluxes $\Lambda \nabla \varphi_{n} \cdot \mathbf{n}$ are clearly continuous through the interfaces of $P_{\Omega}$ since they vanish on either side of each such interface $\partial \Omega_{i} \cap \partial \Omega_{j}$.

To conclude the proof, it remains to find the convenient approximations $\left\{\varphi_{n}^{i}\right\}_{n \geq 1}$ of $\psi \in C_{c, S}^{\infty}(\Omega)$ on $\overline{\Omega_{i}}$.

(iii) Approximation on $\overline{\Omega_{i}}$. Let $\psi \in C_{c, S}^{\infty}(\Omega)$ and let $\mathcal{O}$ be an open set containing $S$ s.t. $\psi \equiv 0$ on a neighborhood of $\overline{\mathcal{O}}$. Let $\left(F_{l}\right)_{1 \leq l \leq r}$ be the faces of $\Omega_{i}$ (i.e. the affine parts of $\partial \Omega_{i}$ of dimension $d-1$ ); for all $1 \leq l \leq r$, we denote by $\mathbf{n}_{l}$ the unit normal to $F_{l}$ pointing inside $\Omega_{i}$ and we define the $C^{2}$ function $f_{l}: \mathbb{R} \times F_{l} \rightarrow \mathbb{R}^{d}$ by

$$
\forall t \in \mathbb{R}, \forall y \in F_{l}, f_{l}(t, y)=y+t \Lambda(y) \mathbf{n}_{l} .
$$

If $(t, y) \in \mathbb{R} \times F_{l}$ and $\left(t^{\prime}, y^{\prime}\right) \in \mathbb{R} \times F_{l}$ are s.t. $f_{l}(t, y)=f_{l}\left(t^{\prime}, y^{\prime}\right)$ then, since $\left(y-y^{\prime}\right) \cdot \mathbf{n}_{l}=0$, one has $t \Lambda(y) \mathbf{n}_{l} \cdot \mathbf{n}_{l}=$ $t^{\prime} \Lambda\left(y^{\prime}\right) \mathbf{n}_{l} \cdot \mathbf{n}_{l}$ and thus

$$
y-y^{\prime}=t \Lambda(y) \mathbf{n}_{l} \cdot \mathbf{n}_{l}\left(\frac{\Lambda\left(y^{\prime}\right) \mathbf{n}_{l}}{\Lambda\left(y^{\prime}\right) \mathbf{n}_{l} \cdot \mathbf{n}_{l}}-\frac{\Lambda(y) \mathbf{n}_{l}}{\Lambda(y) \mathbf{n}_{l} \cdot \mathbf{n}_{l}}\right)
$$

Letting $\varepsilon>0$ be smaller than the inverse of the Lipschitz constant of $y \rightarrow \beta_{0} \frac{\Lambda(y) \mathbf{n}_{l}}{\Lambda(y) \mathbf{n}_{l} \cdot \mathbf{n}_{l}}$ (which is well-defined since $\Lambda(y) \mathbf{n}_{l} \cdot \mathbf{n}_{l}>\alpha_{0}$ for all $\left.y\right)$, (A.2) can happen with $y \neq y^{\prime}$ only if $|t| \geq \varepsilon$. Hence, $f_{l}$ is one-to-one on $(-\varepsilon, \varepsilon) \times F_{l}$. We also notice that $\Lambda(y) \mathbf{n}_{l}$ is uniformly transverse to the hyperplane $H_{l}$ containing $F_{l}$ (this is again $\Lambda(y) \mathbf{n}_{l} \cdot \mathbf{n}_{l} \geq \alpha_{0}$ ) and thus that, upon reducing $\varepsilon$, the Jacobian matrix of $f_{l}$ at any $(t, y) \in(-\varepsilon, \varepsilon) \times F_{l}$ is invertible.

Let $\mathcal{V}_{l}$ be an open neighborhood of $\overline{F_{l} \backslash \mathcal{O}}$ in $F_{l}$ s.t. $\operatorname{dist}\left(\mathcal{V}_{l}, S\right)>0$; the preceding reasoning shows that $f_{l}$ is a $C^{2}$-diffeomorphism from $(-\varepsilon, \varepsilon) \times \mathcal{V}_{l}$ to $f_{l}\left((-\varepsilon, \varepsilon) \times \mathcal{V}_{l}\right)$, an open set in $\mathbb{R}^{d}$ containing in particular $f_{l}\left(\{0\} \times \overline{F_{l} \backslash \mathcal{O}}\right)=\overline{F_{l} \backslash \mathcal{O}}$. Since $\Lambda(y) \mathbf{n}_{l}$ points inside $\Omega_{i}$ (one more time, $\Lambda(y) \mathbf{n}_{l} \cdot \mathbf{n}_{l}>0$ ) and $\operatorname{dist}\left(\mathcal{V}_{l}, S\right)>0$, upon reducing again $\varepsilon$ if needed, we also see that $\mathcal{U}_{l} \stackrel{\text { def }}{=} f_{l}\left(\left[0, \varepsilon\left[\times \mathcal{V}_{l}\right)\right.\right.$ is contained in $\overline{\Omega_{i}}$ and is a neighborhood 


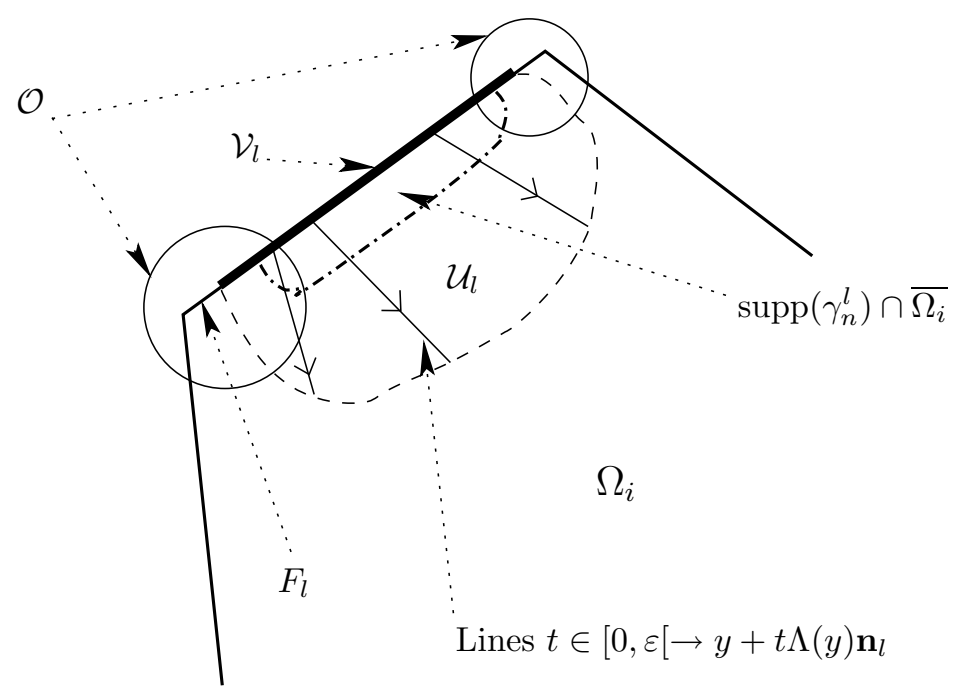

FiguRE 8. Various sets appearing in the proof of Lemma 3.2.

of $\overline{F_{l} \backslash \mathcal{O}}$ in $\overline{\Omega_{i}}$ (see Fig. 8 for a representation of some sets appearing in this proof). Moreover, for all $x \in \mathcal{U}_{l}$, if $x=f_{l}(t, y)$ for $(t, y) \in\left[0, \varepsilon\left[\times \mathcal{V}_{l}\right.\right.$ then $\operatorname{dist}\left(x, H_{l}\right)=(x-y) \cdot \mathbf{n}_{l}=t \Lambda(y) \mathbf{n}_{l} \cdot \mathbf{n}_{l}$ and thus $0 \leq t \leq \frac{1}{\alpha_{0}} \operatorname{dist}\left(x, H_{l}\right)$. This shows that

$$
\forall x \in \mathcal{U}_{l}, \quad \text { if }(t, y)=\left(f_{l \mid\left[0, \varepsilon\left[\times \mathcal{V}_{l}\right.\right.}\right)^{-1}(x) \text { then }|x-y| \leq \frac{\beta_{0}}{\alpha_{0}} \operatorname{dist}\left(x, H_{l}\right) .
$$

Let us define $\psi_{l}$ on $\mathcal{U}_{l}$ s.t.

$$
\psi_{l}\left(f_{l}(t, y)\right)=\psi(y) \text { for all }(t, y) \in\left[0, \varepsilon\left[\times \mathcal{V}_{l}\right.\right.
$$

$\psi_{l}$ belongs to $C^{2}\left(\mathcal{U}_{l}\right)$ and $\psi_{l}=\psi$ on $\mathcal{V}_{l}$ (because $f_{l}(0, y)=y$ ); derivating (A.4) with respect to $t$, taking $t=0$ and using (A.1) we also have

$$
0=\frac{\mathrm{d}}{\mathrm{d} t}\left(\psi_{l}\left(f_{l}(t, y)\right)\right)_{\mid t=0}=\nabla \psi_{l}(y) \cdot \Lambda(y) \mathbf{n}_{l}=\Lambda(y) \nabla \psi_{l}(y) \cdot \mathbf{n}_{l} \quad \text { for all } y \in \mathcal{V}_{l}
$$

As $\psi$ vanishes on a neighborhood of $\overline{\mathcal{O}}$, there exists a neighborhood $\mathcal{N}_{l}$ of $\mathcal{V}_{l} \cap \mathcal{O}$ in $\mathcal{V}_{l}$ s.t. $\psi=0$ on $\mathcal{N}_{l} ;($ A.4) then implies $\psi_{l}=0$ on $f_{l}\left(\left[0, \varepsilon\left[\times \mathcal{N}_{l}\right)\right.\right.$ which is, $f_{l}$ being a diffeomorphism, a neighborhood in $\mathcal{U}_{l}$ of $f_{l}\left(\{0\} \times\left(\mathcal{V}_{l} \cap \mathcal{O}\right)\right)=$ $\mathcal{V}_{l} \cap \mathcal{O} ;$ to sum up,

$$
\psi_{l}=0 \text { on a neighborhood of } \mathcal{V}_{l} \cap \mathcal{O} \text { in } \mathcal{U}_{l} .
$$

For $1 \leq l \leq r$, we take a sequence $\gamma_{n}^{l} \in C_{c}^{\infty}\left(\mathbb{R}^{d} ;[0,1]\right)$ s.t., for all $n \in \mathbb{N}, \gamma_{n}^{l} \equiv 1$ on a neighborhood of $\overline{F_{l} \backslash \mathcal{O}}$ and

$$
\gamma_{n}^{l} \equiv 0 \text { on }\left\{x \in \mathbb{R}^{d}, \operatorname{dist}\left(x, F_{l} \backslash \mathcal{O}\right) \geq 1 / n\right\} \quad \text { and } \quad\left\|\nabla \gamma_{n}^{l}\right\|_{L^{\infty}\left(\mathbb{R}^{d}\right)} \leq C_{13} n
$$

with $C_{13}$ not depending on $n$. If $n$ is large, $\operatorname{supp}\left(\gamma_{n}^{l}\right) \cap \overline{\Omega_{i}}$ is a compact subset of $\mathcal{U}_{l}$ and $\gamma_{n}^{l} \psi_{l}$ can therefore be extended to $\overline{\Omega_{i}}$ by 0 outside $\mathcal{U}_{l}$ without loosing smoothness; we then define $\varphi_{n}=\sum_{l=1}^{r} \gamma_{n}^{l} \psi_{l}+\left(1-\sum_{l=1}^{r} \gamma_{n}^{l}\right) \psi \in$ $C^{2}\left(\overline{\Omega_{i}}\right)$. Since $\psi_{l}=\psi$ on $\mathcal{V}_{l}$ and, for $n$ large enough, $\gamma_{n}^{l}$ vanishes on $\partial \Omega_{i}$ outside $\mathcal{V}_{l}$, we have $\varphi_{n}=\psi$ on $\partial \Omega_{i}$ for such $n$. Still considering large $n$, on a neighborhood of $\overline{F_{l} \backslash \mathcal{O}}$ in $\overline{\Omega_{i}}$ we have $\gamma_{n}^{l}=1$ and $\gamma_{n}^{k}=0$ if $k \neq l$ and therefore, on such a neighborhood, $\varphi_{n}=\psi_{l}$; (A.5) thus shows that $\Lambda \nabla \varphi_{n} \cdot \mathbf{n}=0$ on $\cup_{l=1}^{r} F_{l} \backslash \mathcal{O}=\partial \Omega_{i} \backslash \mathcal{O}$; since all the $\gamma_{n}^{l} \psi_{l}$ and $\psi$ vanish on a neighborhood of $\partial \Omega_{i} \cap \mathcal{O}$ in $\overline{\Omega_{i}}$ (see (A.6)), we obviously also have $\Lambda \nabla \varphi_{n} \cdot \mathbf{n}=0$ on $\partial \Omega_{i} \cap \mathcal{O}$, and thus on the whole boundary of $\Omega_{i}$. It remains to prove that $\varphi_{n} \rightarrow \psi$ in $H^{1}\left(\Omega_{i}\right)$ as $n \rightarrow \infty$; 
in order to achieve this, we write $\varphi_{n}-\psi=\sum_{l=1}^{r} \gamma_{n}^{l}\left(\psi_{l}-\psi\right)$ and use (A.3), (A.4) and the smoothness of $\psi$ to see that, if $\operatorname{dist}\left(x, F_{l} \backslash \mathcal{O}\right) \leq 1 / n$, then $\left|\psi_{l}(x)-\psi(x)\right| \leq C_{14} / n$ with $C_{14}$ not depending on $n$ or $x$ (because $x=f_{l}(t, y)$ with $y \in \mathcal{V}_{l}$ s.t. $\left.|x-y| \leq \beta_{0} /\left(\alpha_{0} n\right)\right)$; we infer from (A.7) that

$$
\left\|\gamma_{n}^{l}\left(\psi_{l}-\psi\right)\right\|_{L^{2}\left(\Omega_{i}\right)} \leq \frac{C_{14}}{n} \operatorname{meas}\left(\Omega_{i}\right)^{1 / 2}
$$

and

$$
\left\|\nabla\left(\gamma_{n}^{l}\left(\psi_{l}-\psi\right)\right)\right\|_{L^{2}\left(\Omega_{i}\right)} \leq C_{13} C_{14} \operatorname{meas}\left(\Omega_{i} \cap \operatorname{supp}\left(\gamma_{n}^{l}\right)\right)^{1 / 2}+\left\|\nabla\left(\psi_{l}-\psi\right)\right\|_{L^{2}\left(\Omega_{i} \cap \operatorname{supp}\left(\gamma_{n}^{l}\right)\right)} .
$$

Since meas $\left(\Omega_{i} \cap \operatorname{supp}\left(\gamma_{n}^{l}\right)\right) \rightarrow 0$ as $n \rightarrow \infty$, this concludes the proof that $\varphi_{n} \rightarrow \psi$ in $H^{1}\left(\Omega_{i}\right)$.

Remark A.1. The proof shows that $\Lambda$ need not be $C^{2}$ on the whole of each $\overline{\Omega_{i}}$, only on the affine parts of $\partial \Omega_{i}$ (and the reader can check that the rest of the paper only requires the $C^{1}$ regularity of $\Lambda$ on each $\overline{\Omega_{i}}$ ).

\section{B. Proof of Lemma 3.3}

Proposition B.1. Let $\mathcal{D}$ be a generic element of a family of discretizations satisfying Assumption 2.1. Let $\varphi \in \mathcal{Q}, \sigma \in \mathcal{E}_{\text {int }}$ with $\mathcal{T}_{\sigma}=\{K, L\}$ and $y_{\sigma} \in \sigma$. Then $\nabla \varphi\left(x_{K}\right)-\nabla \varphi\left(x_{L}\right)$ can be decomposed as follows:

$$
\nabla \varphi\left(x_{K}\right)-\nabla \varphi\left(x_{L}\right)=\mu_{\sigma} \mathbf{n}_{K, \sigma}+\tau_{\sigma} \mathbf{t}_{\sigma}
$$

where $\left|\mathbf{t}_{\sigma}\right|=1, \mathbf{t}_{\sigma} \cdot \mathbf{n}_{K, \sigma}=0$ and the reals $\mu_{\sigma}, \tau_{\sigma}$ verify

$$
\begin{gathered}
\left|\tau_{\sigma}\right| \leq C_{15}[\operatorname{diam}(L)+\operatorname{diam}(K)], \\
\mu_{\sigma}=-\frac{W_{K}\left(x_{L}\right)}{d_{L, \sigma}}+\tau_{\sigma} \frac{\mathbf{t}_{\sigma} \cdot\left(y_{\sigma}-x_{L}\right)}{d_{L, \sigma}}+\frac{W_{K}\left(y_{\sigma}\right)-W_{L}\left(y_{\sigma}\right)}{d_{L, \sigma}},
\end{gathered}
$$

with

$$
W_{K}(x) \stackrel{\text { def }}{=} \varphi(x)-\varphi\left(x_{K}\right)-\nabla \varphi\left(x_{K}\right) \cdot\left(x-x_{K}\right)
$$

and $C_{15} \stackrel{\text { def }}{=} \max \left(\left|\varphi^{\prime \prime}\right|_{L^{\infty}(K)},\left|\varphi^{\prime \prime}\right|_{L^{\infty}(L)}\right)$.

Proof. The vector $\mathbf{t}_{\sigma}$ is obviously the normed orthogonal projection of $\nabla \varphi\left(x_{K}\right)-\nabla \varphi\left(x_{L}\right)$ on the hyperplane parallel to $\sigma$, and the reals $\mu_{\sigma}, \tau_{\sigma}$ are given by the formulæ

$$
\mu_{\sigma}=\left(\nabla \varphi\left(x_{K}\right)-\nabla \varphi\left(x_{L}\right)\right) \cdot \mathbf{n}_{K, \sigma}, \quad \tau_{\sigma}=\left(\nabla \varphi\left(x_{K}\right)-\nabla \varphi\left(x_{L}\right)\right) \cdot \mathbf{t}_{\sigma} .
$$

Since

$$
\begin{aligned}
-W_{K}\left(x_{L}\right)+W_{K}\left(y_{\sigma}\right)-W_{L}\left(y_{\sigma}\right)= & -\varphi\left(x_{L}\right)+\varphi\left(x_{K}\right)+\nabla \varphi\left(x_{K}\right) \cdot\left(x_{L}-x_{K}\right) \\
& +\varphi\left(y_{\sigma}\right)-\varphi\left(x_{K}\right)-\nabla \varphi\left(x_{K}\right) \cdot\left(y_{\sigma}-x_{K}\right) \\
& -\varphi\left(y_{\sigma}\right)+\varphi\left(x_{L}\right)+\nabla \varphi\left(x_{L}\right) \cdot\left(y_{\sigma}-x_{L}\right) \\
= & \nabla \varphi\left(x_{K}\right) \cdot\left(x_{L}-y_{\sigma}\right)+\nabla \varphi\left(x_{L}\right) \cdot\left(y_{\sigma}-x_{L}\right) \\
= & \left(\nabla \varphi\left(x_{K}\right)-\nabla \varphi\left(x_{L}\right)\right) \cdot\left(x_{L}-y_{\sigma}\right),
\end{aligned}
$$

we can use (B.1) and the fact that $\left(x_{L}-y_{\sigma}\right) \cdot \mathbf{n}_{K, \sigma}=d_{L, \sigma}$ to re-write $\mu_{\sigma}$ under the form

$$
\mu_{\sigma}=-\frac{W_{K}\left(x_{L}\right)}{d_{L, \sigma}}+\tau_{\sigma} \frac{\mathbf{t}_{\sigma} \cdot\left(y_{\sigma}-x_{L}\right)}{d_{L, \sigma}}+\frac{W_{K}\left(y_{\sigma}\right)-W_{L}\left(y_{\sigma}\right)}{d_{L, \sigma}} .
$$


The face $\sigma$ is completely contained either in one element of the partition $P_{\Omega}$ or in an interface of this partition; using then either the regularity of $\varphi$ inside each element of the partition or the continuity of its tangential derivatives through the interfaces of $P_{\Omega}$, we can re-write $\tau_{\sigma}$ under the form

$$
\tau_{\sigma}=\left(\nabla \varphi\left(x_{K}\right)-\nabla \varphi\left(y_{\sigma}\right)\right) \cdot \mathbf{t}_{\sigma}+\left(\nabla \varphi\left(y_{\sigma}\right)-\nabla \varphi\left(x_{L}\right)\right) \cdot \mathbf{t}_{\sigma}
$$

and the proof is complete since $\varphi$ is $C^{2}$ on $\bar{K}$ and $\bar{L}$.

Proposition B.2 (flux "quasi-continuity"). Let $\mathcal{D}$ be a generic element of a family of discretizations satisfying Assumption 2.1 and $\varphi \in \mathcal{Q}$. For all $G \in \mathcal{G}, \nabla \varphi\left(x_{K_{G}}\right)$ is the solution of a linear system of equations of the form

$$
\mathcal{A}_{G} Y_{G}=\mathcal{B}_{G}\left(\varphi_{\mathcal{T}}\right)+\mathcal{C}_{G}(\varphi)
$$

where $\varphi_{\mathcal{T}} \in H_{\mathcal{T}}(\Omega)$ is defined by the family $\left\{\varphi\left(x_{K}\right)\right\}_{K \in \mathcal{T}}, \mathcal{A}_{G}$ and $\mathcal{B}_{G}\left(\varphi_{\mathcal{T}}\right)$ are the matrices defined in Lemma 3.1 and the vector $\mathcal{C}_{G}(\varphi)$ verifies

$$
\left|\mathcal{C}_{G}(\varphi)\right| \leq C_{1} \max _{K \in \mathcal{T}_{G}} \operatorname{diam}(K)
$$

with $C_{1}>0$ which only depends on $\varrho_{1}, \varrho_{2}, \Lambda$ and $\varphi$.

Proof. For a cell $K$, let $W_{K}$ be the function defined by (B.4). Since $\varphi$ is $C^{2}$ regular on the closure of each element of $P_{\Omega}$ and since each cell is completely contained in one of these elements, there exists $C_{16}>0$ only depending on $\varphi$ s.t., for all $K \in \mathcal{T}$,

$$
\left|W_{K}(x)\right| \leq C_{16} \operatorname{diam}(K)^{2} \text { for all } x \in \bar{K} .
$$

For all $\sigma \in \mathcal{E}_{\text {int }}$ with $\mathcal{T}_{\sigma}=\{K, L\}$ and $y_{\sigma} \in \sigma$, we apply Proposition B.1 to decompose $\nabla \varphi\left(x_{K}\right)-\nabla \varphi\left(x_{L}\right)($ note that the $W_{K}\left(x_{L}\right)$ appearing in (B.3) is in general not of order 2 with respect to the size of the mesh, since $x_{L} \notin \bar{K}$ and $\varphi$ is not regular across the boundary of some cells). Since $\varphi \in \mathcal{Q}$, we can also write

$$
\frac{1}{\mathrm{~m}_{\sigma}} \int_{\sigma}(\Lambda \nabla \varphi)_{\mid \bar{K}}(x) \cdot \mathbf{n}_{K, \sigma} \mathrm{d} x+\frac{1}{\mathrm{~m}_{\sigma}} \int_{\sigma}(\Lambda \nabla \varphi)_{\mid \bar{L}}(x) \cdot \mathbf{n}_{L, \sigma} \mathrm{d} x=0
$$

and, $\nabla \varphi$ and $\Lambda$ being $C^{1}$ on the closure of each control volume, we deduce from (B.7) that the real $\zeta_{\sigma}(\varphi)=$ $\Lambda_{K} \nabla \varphi\left(x_{K}\right) \cdot \mathbf{n}_{K, \sigma}+\Lambda_{L} \nabla \varphi\left(x_{L}\right) \cdot \mathbf{n}_{L, \sigma}$ verifies

$$
\left|\zeta_{\sigma}(\varphi)\right| \leq C_{17}(\operatorname{diam}(K)+\operatorname{diam}(L))
$$

where $C_{17}>0$ depends only on $\varphi, \Lambda$.

Let us now consider $G \in \mathcal{G}, \sigma \in G \cap \mathcal{E}_{\text {int }}$ and use these preliminary remarks with $K=K_{G}$ and $L$ s.t. $\mathcal{T}_{\sigma}=\left\{K_{G}, L\right\}$. By definition of $\zeta_{\sigma}(\varphi)$ and (B.1),

$$
\begin{aligned}
\left(\Lambda_{L} \mathbf{n}_{L, \sigma}+\Lambda_{K_{G}} \mathbf{n}_{K_{G}, \sigma}\right) \cdot \nabla \varphi\left(x_{K_{G}}\right) & =\Lambda_{L} \mathbf{n}_{L, \sigma} \cdot \nabla \varphi\left(x_{K_{G}}\right)-\Lambda_{L} \mathbf{n}_{L, \sigma} \cdot \nabla \varphi\left(x_{L}\right)+\zeta_{\sigma}(\varphi) \\
& =-\Lambda_{L} \mathbf{n}_{L, \sigma} \cdot \mathbf{n}_{L, \sigma} \mu_{\sigma}+\tau_{\sigma} \Lambda_{L} \mathbf{n}_{L, \sigma} \cdot \mathbf{t}_{\sigma}+\zeta_{\sigma}(\varphi)
\end{aligned}
$$

Equation (B.3) and the definition of $W_{K_{G}}\left(x_{L}\right)$ then show

$$
\begin{aligned}
\left(\Lambda_{L} \mathbf{n}_{L, \sigma}+\Lambda_{K_{G}} \mathbf{n}_{K_{G}, \sigma}\right) \cdot \nabla \varphi\left(x_{K_{G}}\right)= & \frac{\Lambda_{L} \mathbf{n}_{L, \sigma} \cdot \mathbf{n}_{L, \sigma}}{d_{L, \sigma}}\left(\varphi\left(x_{L}\right)-\varphi\left(x_{K_{G}}\right)\right)-\frac{\Lambda_{L} \mathbf{n}_{L, \sigma} \cdot \mathbf{n}_{L, \sigma}}{d_{L, \sigma}} \nabla \varphi\left(x_{K_{G}}\right) \cdot\left(x_{L}-x_{K_{G}}\right) \\
& +\zeta_{\sigma}(\varphi)-\frac{\Lambda_{L} \mathbf{n}_{L, \sigma} \cdot \mathbf{n}_{L, \sigma}}{d_{L, \sigma}}\left(\tau_{\sigma} \mathbf{t}_{\sigma} \cdot\left(y_{\sigma}-x_{L}\right)+W_{K_{G}}\left(y_{\sigma}\right)-W_{L}\left(y_{\sigma}\right)\right) \\
& +\tau_{\sigma} \Lambda_{L} \mathbf{n}_{L, \sigma} \cdot \mathbf{t}_{\sigma}
\end{aligned}
$$


and therefore

$$
\left(\Lambda_{L} \mathbf{n}_{L, \sigma}+\Lambda_{K_{G}} \mathbf{n}_{K_{G}, \sigma}+\frac{\Lambda_{L} \mathbf{n}_{L, \sigma} \cdot \mathbf{n}_{L, \sigma}}{d_{L, \sigma}}\left(x_{L}-x_{K_{G}}\right)\right) \cdot \nabla \varphi\left(x_{K_{G}}\right)=\frac{\Lambda_{L} \mathbf{n}_{L, \sigma} \cdot \mathbf{n}_{L, \sigma}}{d_{L, \sigma}}\left(\varphi\left(x_{L}\right)-\varphi\left(x_{K_{G}}\right)\right)+c_{\sigma}(\varphi)
$$

with $c_{\sigma}(\varphi)=\zeta_{\sigma}(\varphi)-\frac{\Lambda_{L} \mathbf{n}_{L, \sigma} \cdot \mathbf{n}_{L, \sigma}}{d_{L, \sigma}}\left(\tau_{\sigma} \mathbf{t}_{\sigma} \cdot\left(y_{\sigma}-x_{L}\right)+W_{K_{G}}\left(y_{\sigma}\right)-W_{L}\left(y_{\sigma}\right)\right)+\Lambda_{L} \mathbf{n}_{L, \sigma} \cdot \mathbf{t}_{\sigma} \tau_{\sigma}$. If $\sigma \in G \cap \mathcal{E}_{\text {ext }}$, using the definition of $W_{K_{G}}\left(x_{\sigma}\right)$, we have

$$
\frac{\Lambda_{K_{G}} \mathbf{n}_{K_{G}, \sigma} \cdot \mathbf{n}_{K_{G}, \sigma}}{d_{K_{G}, \sigma}} \nabla \varphi\left(x_{K_{G}}\right) \cdot\left(x_{\sigma}-x_{K_{G}}\right)=\frac{\Lambda_{K_{G}} \mathbf{n}_{K_{G}, \sigma} \cdot \mathbf{n}_{K_{G}, \sigma}}{d_{K_{G}, \sigma}}\left(-\varphi\left(x_{K_{G}}\right)\right)+c_{\sigma}(\varphi)
$$

with $c_{\sigma}(\varphi)=-\frac{\Lambda_{K_{G}} \mathbf{n}_{K_{G}, \sigma} \cdot \mathbf{n}_{K_{G}, \sigma}}{d_{K_{G}, \sigma}} W_{K_{G}}\left(x_{\sigma}\right)$.

We deduce that $\nabla \varphi\left(x_{K_{G}}\right)$ is the solution of the linear system of equations

$$
\mathcal{A}_{G} Y_{G}=\mathcal{B}_{G}\left(\varphi_{\mathcal{T}}\right)+\mathcal{C}_{G}(\varphi)
$$

where $\mathcal{C}_{G}(\varphi)$ is the vector of $\mathbb{R}^{d}$ defined by $\left\{c_{\sigma}(\varphi)\right\}_{\sigma \in G}$. Thanks to (B.8), (B.2) and (B.6), there exists $C_{18}>0$ which only depends on $\varrho_{1}, \varrho_{2}, \Lambda$ and $\varphi$ s.t., for all $\sigma \in G$ with $\mathcal{T}_{\sigma}=\left\{K_{G}, L\right\},\left|c_{\sigma}(\varphi)\right| \leq C_{18}(\operatorname{diam}(L)+$ $\left.\operatorname{diam}\left(K_{G}\right)\right)$. The proof is complete.

We are now in a position to prove Lemma 3.3. Let $W_{K}$ the function defined by (B.4) and recall that (B.6) holds. Since $\left(\nabla_{\mathcal{D}} \varphi_{\mathcal{T}}\right)_{K_{G}}^{G}$ is the solution of the linear system (3.3) with $v=\varphi_{\mathcal{T}}$, we can deduce from Proposition B.2 that $\nabla \varphi\left(x_{K_{G}}\right)-\left(\nabla_{\mathcal{D}} \varphi_{\mathcal{T}}\right)_{K_{G}}^{G}$ is the solution of the linear system $\mathcal{A}_{G} Z_{G}=\mathcal{C}_{G}(\varphi)$ where the vector $\mathcal{C}_{G}(\varphi)$ satisfies (B.5). We obtain

$$
\left|\nabla \varphi\left(x_{K_{G}}\right)-\left(\nabla_{\mathcal{D}} \varphi_{\mathcal{T}}\right)_{K_{G}}^{G}\right| \leq C_{1}\left|\mathcal{A}_{G}^{-1}\right| \max _{K \in \mathcal{T}_{G}} \operatorname{diam}(K) .
$$

For all $\sigma \in G \cap \mathcal{E}_{\text {int }}$ with $\mathcal{T}_{\sigma}=\left\{K_{G}, L\right\}$, thanks to (3.4) with $v=\varphi_{\mathcal{T}}$, we have

$$
\left(\nabla_{\mathcal{D}} \varphi_{\mathcal{T}}\right)_{L}^{G, \sigma}=\left(\nabla_{\mathcal{D}} \varphi_{\mathcal{T}}\right)_{K_{G}}^{G}-\frac{R_{L, \sigma}\left(\varphi_{\mathcal{T}}\right)}{d_{L, \sigma}} \mathbf{n}_{L, \sigma}
$$

where $R_{L, \sigma}\left(\varphi_{\mathcal{T}}\right)=\varphi\left(x_{L}\right)-\varphi\left(x_{K_{G}}\right)-\left(\nabla_{\mathcal{D}} \varphi_{\mathcal{T}}\right)_{K_{G}}^{G} \cdot\left(x_{L}-x_{K_{G}}\right)$. Thanks to Proposition B.1, we can deduce that

$$
\begin{aligned}
\nabla \varphi\left(x_{L}\right)-\left(\nabla_{\mathcal{D}} \varphi_{\mathcal{T}}\right)_{L}^{G, \sigma}= & \nabla \varphi\left(x_{K_{G}}\right)+\mu_{\sigma} \mathbf{n}_{L, \sigma}-\tau_{\sigma} \mathbf{t}_{\sigma}-\left(\nabla_{\mathcal{D}} \varphi_{\mathcal{T}}\right)_{K_{G}}^{G}+\frac{R_{L, \sigma}\left(\varphi_{\mathcal{T}}\right)}{d_{L, \sigma}} \mathbf{n}_{L, \sigma} \\
= & \nabla \varphi\left(x_{K_{G}}\right)-\left(\nabla_{\mathcal{D}} \varphi_{\mathcal{T}}\right)_{K_{G}}^{G}+\frac{R_{L, \sigma}\left(\varphi_{\mathcal{T}}\right)-W_{K_{G}}\left(x_{L}\right)}{d_{L, \sigma}} \mathbf{n}_{L, \sigma} \\
& +\left(\tau_{\sigma} \frac{\mathbf{t}_{\sigma} \cdot\left(y_{\sigma}-x_{L}\right)}{d_{L, \sigma}}+\frac{W_{K_{G}}\left(y_{\sigma}\right)-W_{L}\left(y_{\sigma}\right)}{d_{L, \sigma}}\right) \mathbf{n}_{L, \sigma}-\tau_{\sigma} \mathbf{t}_{\sigma} \\
= & \nabla \varphi\left(x_{K_{G}}\right)-\left(\nabla_{\mathcal{D}} \varphi_{\mathcal{T}}\right)_{K_{G}}^{G}+\left(\nabla \varphi\left(x_{K_{G}}\right)-\left(\nabla_{\mathcal{D}} \varphi_{\mathcal{T}}\right)_{K_{G}}^{G}\right) \cdot \frac{\left(x_{L}-x_{K_{G}}\right)}{d_{L, \sigma}} \mathbf{n}_{L, \sigma} \\
& +\left(\tau_{\sigma} \frac{\mathbf{t}_{\sigma} \cdot\left(y_{\sigma}-x_{L}\right)}{d_{L, \sigma}}+\frac{W_{K_{G}}\left(y_{\sigma}\right)-W_{L}\left(y_{\sigma}\right)}{d_{L, \sigma}}\right) \mathbf{n}_{L, \sigma}-\tau_{\sigma} \mathbf{t}_{\sigma} .
\end{aligned}
$$

Using then (B.9), (B.6) and (2.2), we can deduce that there exists a real $C_{19}>0$ which only depends on $\varrho_{1}, \varrho_{2}$, $\Lambda$ and $\varphi$ s.t.

$$
\left|\nabla \varphi\left(x_{L}\right)-\left(\nabla_{\mathcal{D}} \varphi_{\mathcal{T}}\right)_{L}^{G, \sigma}\right| \leq C_{19}\left(1+\left|\mathcal{A}_{G}^{-1}\right|\right) \max _{K \in \mathcal{T}_{G}} \operatorname{diam}(K),
$$

and the proof is complete. 


\section{REFERENCES}

[1] I. Aavatsmark, An introduction to multipoint flux approximations for quadrilateral grids. Comput. Geosci. 6 (2002) 405-432.

[2] I. Aavatsmark, T. Barkve, $\varnothing$. Bøe and T. Mannseth, Discretization on non-orthogonal, curvilinear grids for multi-phase flow, in Proc. of the 4th European Conf. on the Mathematics of Oil Recovery (Røros, Norway), Vol. D (1994).

[3] I. Aavatsmark, G.T. Eigestad, B.T. Mallison, J.M. Nordbotten and E. Øian, A new finite volume approach to efficient discretization on challeging grids, in Proc. SPE 106435, Houston, USA (2005).

[4] I. Aavatsmark, G.T. Eigestad, R.A. Klausen, M.F. Wheeler and I. Yotov, Convergence of a symmetric MPFA method on quadrilateral grids. Comput. Geosci. 11 (2007) 333-345.

[5] I. Aavatsmark, G.T. Eigestad, B.T. Mallison and J.M. Nordbotten, A compact multipoint flux approximation method with improved robustness. Numer. Methods Partial Differ. Equ. 24 (2008) 1329-1360.

[6] L. Agélas and D.A. Di Pietro, A symmetric finite volume scheme for anisotropic heterogeneous second-order elliptic problems, in Finite Volumes for Complex Applications, V.R. Eymard and J.-M. Hérard Eds., John Wiley \& Sons (2008) 705-716.

[7] L. Agélas and R. Masson, Convergence of the finite volume MPFA O scheme for heterogeneous anisotropic diffusion problems on general meshes. C. R. Acad. Sci. Paris, Sér. I 346 (2008) 1007-1012.

[8] L. Agélas and R. Masson, Convergence of finite volume MPFA O type schemes for heterogeneous anisotropic diffusion problems on general meshes. Preprint available at http://hal.archives-ouvertes.fr/hal-00340159/fr (2008).

[9] L. Agélas, D.A. Di Pietro and R. Masson, A symmetric and coercive finite volume scheme for multiphase porous media flow with applications in the oil industry, in Finite Volumes for Complex Applications, V.R. Eymard and J.-M. Hérard Eds., John Wiley \& Sons (2008) 35-52.

[10] L. Agélas, D.A. Di Pietro, R. Eymard and R. Masson, An abstract analysis framework for nonconforming approximations of diffusion problems on general meshes. IJFV 7 (2010) 1-29.

[11] S. Balay, W.D. Gropp, L.C. McInnes and B.F. Smith, Efficient management of parallelism in object oriented numerical software libraries, in Modern Software Tools in Scientific Computing, E. Arge, A.M. Bruaset and H.P. Langtangen Eds., Birkhäuser Press (1997) 163-202.

[12] S. Balay, K. Buschelman, W.D. Gropp, D. Kaushik, M.G. Knepley, L.C. McInnes, B.F. Smith and H. Zhang, PETSc Web page (2001) www.mcs.anl.gov/petsc.

[13] S. Balay, K. Buschelman, V. Eijkhout, W.D. Gropp, D. Kaushik, M.G. Knepley, L.C. McInnes, B.F. Smith and H. Zhang, PETSc users manual. Tech. Report ANL-95/11 - Revision 2.1.5, Argonne National Laboratory (2004).

[14] F. Brezzi, K. Lipnikov and M. Shashkov, Convergence of mimetic finite difference methods for diffusion problems on polyhedral meshes. SIAM J. Numer. Anal. 45 (2005) 1872-1896.

[15] F. Brezzi, K. Lipnikov and V. Simoncini, A family of mimetic finite difference methods on polygonal and polyhedral meshes. Math. Mod. Meths. Appli. Sci. 15 (2005) 1533-1553.

[16] F. Brezzi, K. Lipnikov and M. Shashkov, Convergence of mimetic finite difference methods for diffusion problems on polyhedral meshes with curved faces. Math. Mod. Meths. Appli. Sci. 26 (2006) 275-298.

[17] D.A. Di Pietro and A. Ern, Discrete functional analysis tools for discontinuous Galerkin methods with application to the incompressible Navier-Stokes equations. Math. Comp. (2010), preprint available at http://hal.archives-ouvertes.fr/ hal-00278925/fr/.

[18] J. Droniou, A density result in Sobolev spaces. J. Math. Pures Appl. 81 (2002) 697-714.

[19] J. Droniou and R. Eymard, A mixed finite volume scheme for anisotropic diffusion problems on any grid. Numer. Math. 105 (2006) 35-71.

[20] J. Droniou, R. Eymard, T. Gallouët and R. Herbin, A unified approach to Mimetic Finite Difference, Hybrid Finite Volume and Mixed Finite Volume methods. Maths. Models Methods Appl. Sci. 20 (2010) 1-31.

[21] M.G. Edwards and C.F. Rogers, A flux continuous scheme for the full tensor pressure equation, in Proc. of the 4th European Conf. on the Mathematics of Oil Recovery (Røros, Norway), Vol. D (1994).

[22] R. Eymard, T. Gallouët and R. Herbin, The finite volume method, Ph.G. Charlet and J.-L. Lions Eds., North Holland (2000).

[23] R. Eymard, R. Herbin and J.C. Latché, Convergence analysis of a colocated finite volume scheme for the incompressible Navier-Stokes equations on general 2D or 3D meshes. SIAM J. Numer. Anal. 45 (2007) 1-36.

[24] R. Eymard, T. Gallouët and R. Herbin, Discretization of heterogeneous and anisotropic diffusion problems on general non-conforming meshes. SUSHI: a scheme using stabilization and hybrid interfaces. IMA J. Numer. Anal. (2009) doi: 10.1093/imanum/drn084.

[25] M. Vohralík, Equivalence between lowest-order mixed finite element and multi-point finite volume methods on simplicial meshes. ESAIM: M2AN 40 (2006) 367-391. 